A Performance Evaluation of Pointer-Based Joins \#

by

Eugene J. Shekita

Michael J. Carey

Computer Sciences Technical Report \#916

March 1990 



\title{
A Performance Evaluation of Pointer-Based Joins
}

\author{
Eugene J. Shekita \\ Michael J. Carey
University of Wisconsin
Madison, WI 53706 \\ Computer Sciences Department
}

This research was partially supported by the Defense Advanced Research Projects Agency under contract N00014-85-K-0788, by the National Science Foundation under grant IRI-8657323, by DEC through its Incentives for Excellence program, and by a donation from Texas Instruments. 


\title{
A Performance Evaluation of Pointer-Based Joins
}

\author{
Eugene J. Shekita \\ Michael J. Carey \\ Computer Sciences Department \\ University of Wisconsin \\ Madison, WI 53706
}

\begin{abstract}
In this paper we describe three pointer-based join algorithms that are simple variants of the nested-loops, sort-merge, and hybrid-hash join algorithms used in relational database systems. Each join algorithm is described and an analysis is carried out to compare the performance of the pointer-based algorithms to their standard, non-pointer-based counterparts. The results of the analysis show that the pointer-based algorithms can provide significant performance gains in many situations. The results also show that the pointer-based nested-loops join algorithm, which is perhaps the most natural pointer-based join algorithm to consider using in an objectoriented database system, performs quite poorly on most medium to large joins.
\end{abstract}

\section{INTRODUCTION}

Most semantic and object-oriented data models include the notion of reference attributes or object-valued attributes (e.g., [Ship81, Zani83, Care88, Kim89, Deux90]). Semantic and object-oriented database systems typically implement such attributes with pointers or object identifiers (OIDs). OIDs can be purely logical, but often they have a physical component [Chan82, Care89, Vele89, Deux90]. Relational database systems also support the notion of physical pointers in the form of record identifiers (RIDs). These are generally used to implement indexes and various internal data structures, but they can also be used to provide efficient support for referential integrity and certain kinds of joins [Care90, Haas90]. In view of the fact that physical pointers are supported by a variety of database systems, it seems appropriate to examine how such pointers can be used in query processing.

In this paper we describe how pointers can be used to process join queries. We describe three pointer-based join algorithms that are simple variants of the well known nested-loops, sort-merge, and hybrid-hash join algorithms [Blas77, Shap86]. The algorithms we describe can be utilized in any database system where physical pointers are used to link records or objects together. In a relational system, our algorithms would presumably be used to process foreign-key joins whenever the relations being joined are linked internally by RIDs to provide support for referential integrity or pointer-based joins (e.g., as described in [Care90, Haas90]). And in semantic and object-oriented database systems, our algorithms would presumably be used to process functional joins, that is, joins between sets that are linked together by object-valued attributes or OIDs. 
The remainder of this paper examines the three pointer-based join algorithms mentioned above in some detail. Each algorithm is described, and then an analytical model is employed to compare the performance of the pointerbased algorithms to their standard, non-pointer-based counterparts. Results are presented for large, full-relation joins and small to medium-sized joins with a selection predicate.

The rest of this paper is organized as follows: Section 2 illustrates the type of joins that will be analyzed and provides examples of how such joins arise in relational and object-oriented database systems. In Section 3, descriptions of the join algorithms that will be analyzed are given. The pointer-based join algorithms that are described assume a simple many-to-one pointer structure. In Section 4, the pointer-based algorithms are then analyzed and compared to their standard, non-pointer-based counterparts. In Section 5, two other pointer-based join algorithms for use with a one-to-many pointer structure are described. The analysis of those algorithms is left for future work. Finally, in Section 6, related work is briefly mentioned, and conclusions are drawn in Section 7.

\section{THE TYPES OF JOINS THAT ARE ANALYZED}

In this section, we provide simple examples of the types of joins that will be analyzed. The examples are intended to show that there are indeed practical situations in which pointer-based join algorithms can be used, both in relational database systems and in object-oriented database systems. At the end of this section, we also note the types of joins which are not amenable to the pointer-based algorithms that are analyzed.

Throughout this paper, we will be concerned with the join of two sets, denoted $\mathrm{R}$ and $\mathrm{S}$, that stand in a manyto-one relationship with each other. We will assume a simple pointer structure, where each object in $\mathrm{R}$ contains a pointer to its related object in S. Although pointer-based join algorithms can be developed for other relationships with different pointer structures, this is perhaps the most natural one to consider initially.

\subsection{Pointer-Based Joins in a Relational Database System}

In a relational context, the types of joins being considered are illustrated by the following schema:

Emp (name: char[ ], age: int, jobid: int ...)

Job (jobid: int, name: char[ ], wage: int ...)

Here, Emp and Job stand in a many-to-one relationship to each other, with the attribute jobid serving as a foreign key for the Job relation. The joins we will be analyzing have the form:

retrieve (Emp.name, Emp.age, Job.name)

where Emp.jobid $=$ Job.jobid

and Emp.age $<30$ 
These sorts of foreign-key joins are probably the most common type of join in a relational database system. They are also a natural candidate for using pointer-based joins. For example, it is fairly easy to imagine a situation where the database system initializes and maintains hidden pointers (i.e., RIDs) in each Emp record, linking it to its associated job record. This is in fact what the incremental join facility in Starburst does (as described in [Care90, Haas90]), and it could also be easily applied to the sort of reference attributes described in [Zani83].

\subsection{Pointer-Based Joins in an Object-Oriented Database System}

In an object-oriented database system, the opportunity for pointer-based joins arises quite naturally because pointers in the form of OIDs are commonly used to implement object-valued attributes. The following schema, which has been written in the EXTRA data model [Care88], illustrates how our example join might appear in an object-oriented system (or in GEM [Zani83] for that matter):

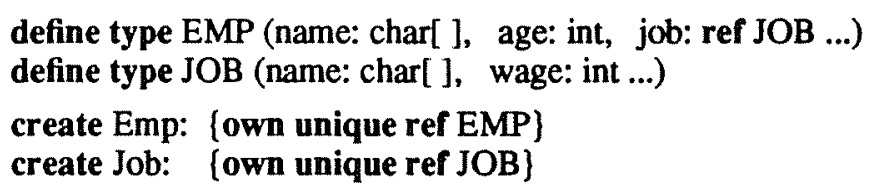

Here, the top half of the schema defines the structure of the objects in the Emp and Job sets that are created in the bottom half of the schema. In the EXTRA data model, the notation own unique ref indicates ownership or an existence dependency; therefore, if the set Emp is deleted, all the employee objects in Emp are also deleted. The key thing to recognize here is that the attribute jobid, which served as the foreign key in the relational schema, has been replaced by the object-valued attribute job, consequently:

\section{retrieve (Emp.name, Emp.age, Emp.job.name)}

where Emp.age $<30$

has the same semantics as the foreign-key join presented above. Depending on the application, these sorts of joins may be more or less common in an object-oriented database system, but we still expect them to represent an important class of queries.

For our pointer-based join algorithms to work in an object-oriented setting, it is necessary for object-valued attributes to be implemented with OIDs that have a physical component (as opposed to strictly logical OIDs). It is also necessary for sets such as Emp and Job to be stored as disk files in much the same way that they would be stored in a relational database system. There are currently at least two object-oriented databases being developed that fulfill these requirements [Care89, Vele89, Deux90]. 


\subsection{The Types of Joins Not Analyzed}

It is important to note that the pointer-based join algorithms which are analyzed in this paper can be used on either (i) full joins or (ii) select-project-join queries in which there is a selection predicate on Emp that is more restrictive than any selection predicate on Job. The pointer-based algorithms that are analyzed cannot, however, be used effectively on select-project-join queries in which the selection predicate on Job is the more restrictive one. This is because the most efficient way to process such joins is in a direction that is opposite to the pointers that link Emp and Job. For example, in a join with a selection predicate on Job and no selection predicate on Emp, the best execution plan is likely to be one in which the selection on Job is evaluated first and then joined with Emp.

To process such joins with pointers, the simple many-to-one pointer structure that we have assumed is inadequate. What is needed is some sort of structure that links each object in Job to the objects in Emp that are related to it. One possibility is the kind of Codasyl-like pointer structure used in [Care90]. Another possibility is a one-tomany pointer structure, where each object in Job contains a set of embedded pointers linking that object to its related objects in Emp. In Section 5, we present two pointer-based join algorithms that use such a pointer structure. Their analysis is left for future work, however.

\section{DESCRIPTIONS OF THE JOIN ALGORITHMS}

This section describes the pointer-based join algorithms that will be analyzed. To be consistent, we shall use object-oriented database terminology throughout this section and in the analysis, but it should be clear how the discussion applies in a relational setting as well.

The description and analysis of the algorithms is based on the join of two object sets, denoted $\mathrm{R}$ and $\mathrm{S}$, which stand in a many-to-one relationship with each other. Both $R$ and $S$ are assumed to be stored as separate disk files. In the standard, non-pointer-based join algorithms, we assume that each object in $\mathrm{R}$ contains a foreign key for the object it references in S, while in the pointer-based join algorithms, we assume that each object in $\mathrm{R}$ contains a pointer to the object it references in $S$.

As indicated earlier, our pointer-based join algorithms assume that physical pointers are used. In particular, we assume that each pointer includes a page identifier or PID that points directly to the disk page of the object that the pointer references. It is unclear whether our pointer-based join algorithms would be worthwhile in system where pointers are logical surrogates that have to be translated via a system-wide table, as in [Horn87]. But as indicated in [Shek88], physical pointers like the ones we are assuming can provide most of the functionality that logical surrogates provide without the overhead of a system-wide translation table.

The remainder of this section describes our pointer-based join algorithms. First, however, their standard, non-pointer-based counterparts are reviewed. Note that only high-level descriptions of the algorithms are given 
here. Details such as how main memory is partitioned are addressed in the analysis. In addition, selections and projections are generally ignored for now. They will also be treated more thoroughly in the analysis.

\subsection{Standard, Non-Pointer-Based Join Algorithms}

\subsubsection{Standard Nested-Loops/Index-Nested-Loops}

The nested-loops join algorithm usually performs poorly on full joins, and consequently it is not considered for such joins in the analysis. It will be used, however, in the analysis of small to medium-sized joins with a selection predicate on R. For those types of joins, a version of the nested-loops algorithm commonly referred to as index-nested-loops is used [Blas77]. Index-nested-loops is an option only when there is an index on S. It executes as follows:

(1) Let $\mathrm{R}^{\prime}$ be the result of the selection on $\mathrm{R}$. The objects in $\mathrm{R}^{\prime}$ are first sorted by their join atribute (i.e., foreign key).

(2) Each object in $R^{\prime}$ is then examined and its join attribute is used to probe the index on $S$ to find the object that it joins with in S.

Note that this is slightly different than the conventional index-nested-loops algorithm in that $\mathrm{R}^{\prime}$ is sorted here. We have chosen this variation because when the index on $\mathrm{S}$ is a $\mathrm{B}+$ tree, and when $\mathrm{R}^{\prime}$ is small, as in the examples we analyze, sorting $\mathrm{R}^{\prime}$ in this manner reduces the cost of probing the index enough to lower the overall cost of the join. More will be said about this in the analysis.

\subsubsection{Standard Sort-Merge}

Although sort-merge has been shown to be generally inferior to hybrid-hash [Dewi85, Shap86], it is still considered in the analysis because it is used by many relational database systems. In the analysis, we will assume that memory is large enough so that $\mathrm{R}$ and $\mathrm{S}$ can be sorted and merged in two passes, as described in [Shap86]. Under that assumption, the algorithm executes as follows:

(1) R is read into memory and sorted output runs are generated using a heap or some other priority queue structure. The output runs are sorted by the value of the join attribute. The same is done for $\mathrm{S}$.

(2) The output runs of $R$ and $S$ are then concurrently merged in memory. As objects from $R$ and $S$ are produced in sorted order by these merges, they are checked to see if their join attributes match. When an object from $R$ matches one from $\mathrm{S}$, the two are joined and the result is output.

\subsubsection{Standard Hybrid-Hash}

Hybrid-hash is usually considered to be the best-performing join algorithm when a full join is performed [Shap86]. Assuming that the size of $\mathrm{S}$ is bigger than $\mathrm{R}$ (otherwise exchange $\mathrm{R}$ and $\mathrm{S}$ ), it executes as follows: 
(1) $\mathrm{R}$ is read into memory ${ }^{1}$ and divided into $B+1$ partitions $R_{0}, R_{1}, \ldots R_{B}$ on the basis of some hash function that is applied to the join attribute. The same is done for $\mathrm{S}$; thus, $R_{i}$ will only join with $S_{i}$ for $0 \leq i \leq B$. The value of $B$ is chosen such that $S_{0}$ can be joined in memory with $R_{0}$ as $S$ is being partitioned and such that a hash table for each $R_{i}$ can later fit in memory for $i \geq 1$.

(2) After $S$ has been partitioned and $S_{0}$ has been joined with $R_{0}$, each $R_{i}$ is joined with $S_{i}$ for $1 \leq i \leq B$.

(3) The joins between $R_{i}$ and $S_{i}$ for $0 \leq i \leq B$ are all performed with hashing. $R_{i}$ is read into memory, and each object in $R_{i}$ is hashed on its join attribute and inserted into a hash table. $S_{i}$ is then read into memory, and the join attribute of each object in $S_{i}$ is used to probe the hash table to find the object that it joins with in $R_{i}$.

In the above description, $\mathrm{R}$ is said to play the role of the inner set, while $\mathrm{S}$ is said to play the role of the outer set. To minimize the number of I/Os, hybrid-hash always chooses the smaller of $\mathrm{R}$ and $\mathrm{S}$ to play the role of the inner set [Shap86].

\subsubsection{Using Bit Filters in Standard Sort-Merge and Hybrid-Hash}

As mentioned earlier, we shall consider small to medium-sized joins with a selection predicate on $R$ in the analysis. For such joins, a technique call bit filtering [Dewi85, Mack86] can be employed to reduce the cost of the sort-merge and hybrid-hash algorithms. In both algorithms, bit filtering works as follows:

(1) Let $R^{\prime}$ be the result of the selection on $R$. Prior to executing the join, a bit vector in memory is initialized by setting all of its bits to zero. Then, as $R^{\prime}$ is read, the join attribute of each object in $R^{\prime}$ is hashed some number of times, and each resulting hash value is used to set a bit in the bit vector.

(2) Later, when $S$ is read, the join attribute of each object in $S$ is hashed in the same manner as in step (1), and the resulting hash values are used to check the bit vector. If one or more of the bits are not set, then that object can be safely discarded since it cannot possibly join with any object in $\mathbf{R}^{\prime}$.

The net effect of bit filtering is to filter out most of the objects in $S$ that do not participate in the join at an early processing stage. As demonstrated in [Dewi85], this can result in significant savings due to the fact that these non-participating objects do not have to be stored on disk between the various phases of the sort-merge and hybridhash algorithms. In the analysis of small to medium-sized joins with a selection predicate on $R$, the analysis of both the sort-merge and hybrid-hash algorithms will include the effects of using a one-page bit filter.

\footnotetext{
1 When we say that "R is read into memory", we mean that $\mathrm{R}$ is incrementally read into memory page by page; that is, page $P_{i+1}$ is read only after all the objects in page $P_{i}$ have been processed. This terminology shall be used throughout the description of the hybrid-hash algorithm.
} 


\subsection{Pointer-Based Join Algorithms}

\subsubsection{Pointer-Based Nested-Loops}

The pointer-based nested-loops algorithm is the algorithm that results when naive pointer traversal is used to compute the join. In the algorithm, only one page of memory is allocated to read $R$, and the rest are allocated to read $S . R$ is read into memory page by page. When a page of $R$ is read into memory, the objects on that page are scanned one by one, and the pointer in each object $r$ that is scanned is used to identify the object $s$ that it joins with in S. The page containing $s$ is read into memory (if it is not already there) and $r$ is joined with $s$.

\subsubsection{Pointer-Based Sort-Merge}

As the analysis will clearly show, one of the problems with the pointer-based nested-loops algorithm is that it makes no attempt to optimize disk reads. As a result, a particular disk page in $S$ can end up being read more than once. For example, suppose that two objects $r_{1}$ and $r_{2}$ reference the same object $s$ in S. Depending on how R is organized, $r_{1}$ and $r_{2}$ may not be physically clustered on the same disk page in $\mathrm{R}$. If that is the case, then between the time when $r_{1}$ is joined to $s$ and the time when $r_{2}$ is joined to $s$, the page $P$ containing $s$ may be paged out of memory by the buffer replacement algorithm. In that event, $P$ would have to be read twice, once to join $r_{1}$ with $s$, and a second time to join $r_{2}$ with $s$.

The pointer-based sort-merge algorithm avoids this problem by first sorting all of the objects in $\mathrm{R}$ by the value of the page identifiers (PIDs) stored in their pointers. The effect of sorting $R$ in this manner is to group all of the objects in $\mathrm{R}$ that reference the same page in $\mathrm{S}$. Doing so guarantees that each page in $\mathrm{S}$ will be read only once. The algorithm executes as follows:

(1) $\mathrm{R}$ is read into memory and sorted much like it is in the standard sort-merge algorithm, except that here the output runs are sorted by PID values ${ }^{2}$ rather than by the join attribute. $S$ is not sorted.

(2) The output runs of $\mathbf{R}$ are then merged in memory. Each object $r$ produced by the merge is examined and its pointer is used to identify the object $s$ that it joins with in S. The page containing $s$ is read into memory and $r$ is joined with $s$.

\subsubsection{Pointer-Based Hybrid-Hash}

The pointer-based hybrid-hash algorithm groups the objects in $R$ by PID values much like the pointer-based sort-merge algorithm. Instead of sorting $\mathrm{R}$, however, hashing is used to group the objects that reference the same page in S. The algorithm executes as follows:

\footnotetext{
${ }^{2}$ In the descriptions of the pointer-based son-merge and hybrid-hash algorithms, "PID value" is meant to be understood as the value of the page identifier that is stored in a pointer.
} 
(1) $\mathrm{R}$ is partitioned much like it is in the standard hybrid-hash algorithm, except that here it is partitioned by PID values rather than by the join attribute. $S$ is not partitioned.

(2) Each partition $R_{i}$ of $\mathrm{R}$ is joined with $\mathrm{S}$ by taking $R_{i}$ and building a hash table for it in memory. The hash table is built by hashing each object $r$ in $R_{i}$ by the value of its pointer's PID. The hash table is built in such a way that all objects which reference the same page in $S$ are grouped together in the same hash entry (see Figure 1).

(3) Once the hash table for $R_{i}$ has been built, each of its hash chains is scanned. Each time a new hash entry $H$ is encountered on a chain, the page in $S$ associated with $H$ is read, and all of the objects in R that reference that page, which have been grouped in $H$, are joined with their corresponding objects in $\mathrm{S}$.

Note that one of the key differences between this algorithm and the standard hybrid-hash algorithm is that $R$ is the only set that is partitioned, and as such it always plays the role of the inner set. This is necessary because the direction of the pointers is from $\mathrm{R}$ to $\mathrm{S}$. As we shall see, when $\mathrm{R}$ is significantly larger than $\mathrm{S}$, this can cause the standard hybrid-hash algorithm to outperform the pointer-based hybrid-hash algorithm.

\section{ANALYZING THE JOIN ALGORITHMS}

In this section, an analysis is performed to quantitatively compare the first set of pointer-based join algorithms to their standard, non-pointer-based counterparts. The net CPU and $\mathrm{I} / \mathrm{O}$ cost of executing each join algorithm is derived and used as the basis for comparison. Two types of joins are analyzed: full joins, where all of $R$ is joined to all of $S$, and small to medium-sized joins, where the result of a selection on $R$ is joined to $S$. For the small to medium-sized joins, the selectivity of the predicate on $\mathrm{R}$ will be varied to control the size of the joins. Projections are not considered in the analysis, since they do not change the general results in any significant way.

\subsection{Assumptions in the Analysis}

Figure 2 depicts the file structure that is assumed in the analysis. The object sets $\mathrm{R}$ and $\mathrm{S}$ are assumed to be stored as separate disk files, with each object in $\mathrm{R}$ referencing its related object in $\mathrm{S}$ via en embedded pointer or

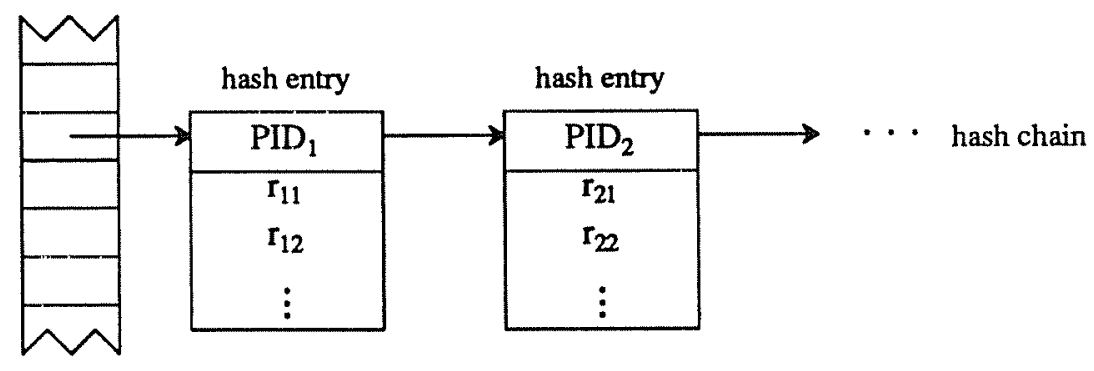

Figure 1: The Structure of the Hash Table for $R_{i}$ 
foreign key.

One of the key assumptions in the analysis is that $\mathrm{R}$ and $\mathrm{S}$ are relatively unclustered. By this we mean that objects in $\mathrm{R}$ are not ordered by their references to $\mathrm{S}$. The reader should bear in mind that this is an important assumption and has a considerable impact on the analysis, since it makes joins between $\mathrm{R}$ and $\mathrm{S}$ more expensive. We make this assumption because we feel that it represents a common case, as the objects in $\mathrm{R}$ would often be ordered by the value of some data field, not by their references to $S$. Another reason for this assumption is because it is the most difficult case to analyze. In fact, using the techniques developed in this section, the analysis for when $\mathrm{R}$ and $\mathrm{S}$ are relatively clustered or clustered together in the same file is straightforward.

For uniformity, and to make the analysis tractable, we assume that each object in $\mathrm{S}$ is referenced by exactly $k$ objects in R. The value $k$ is referred to as the sharing level and it is varied in the analysis. In Figure 2, for example, the value of $k$ is 2 . The result of this assumption is that the cardinality (although not necessarily the size) of S will always be equal to $1 / k$ times the cardinality of $R$. In general, this is an unrealistic assumption because in practice $R$ may reference only part of $\mathrm{S}$. This does not invalidate the analysis, however, because we shall also consider joins where there is a selection predicate on $\mathbf{R}$, and these are effectively like joins in which $\mathbf{R}$ references only part of $\mathbf{S}$.

As Figure 2 illustrates, we will also assume that $B+$ trees exist on both $R$ and $S$. These will be used in the small to medium-sized joins with a selection predicate on $\mathrm{R}$. The index on $\mathrm{R}$ will be used to evaluate the selection predicate, while the index on $\mathrm{S}$ will be used by the index-nested-loops algorithm to avoid a file scan of $S$.

Finally, we will ignore the minimal memory requirements of the different join algorithms in the analysis and simply assume that there is always enough memory for each of the algorithms to execute. In all the examples that are analyzed, the minimal memory requirements turn out to be quite modest, so this is a reasonable assumption to make.

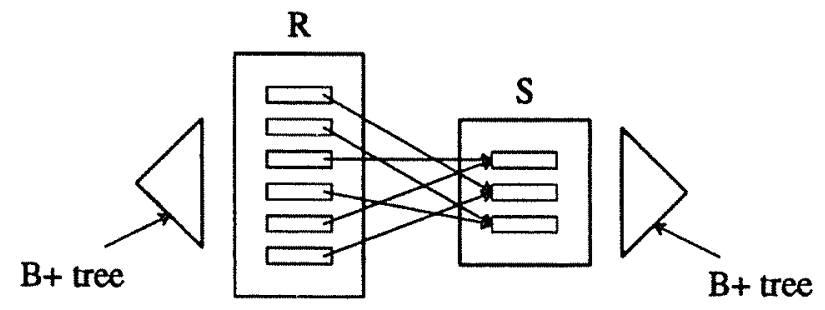

Figure 2: The Reference Structure of $R$ and $S$ 


\subsection{Parameters Used in the Analysis}

The parameters that are used in the analysis are listed in Table 1. Although there are a large number of parameters, only a few of them are actually varied. Moreover, most of the parameters are not really parameters per se, but rather functions of a small set of "core" parameters, which consist of the parameters in the top half of Table 1. Defaults for the core parameters are listed in Table 2.

The meaning of most parameters should be clear from Table 1. The parameters that are kept fixed in the analysis are noted as such in Table 2. As indicated, all times are given in terms of milliseconds. The time to compare, hash, move, and swap values (in memory) has been expressed as a function of the number of instructions executed and the instruction rate of the machine. This has been done because it allows us to look at how different CPU execution rates affect the results. The values for move ${ }_{r}$ and move $e_{s}$ are based on the time to execute a small, 4instruction loop that moves objects in word-size chunks. Finally, the so-called "fudge factor", $F$, which was introduced in [Shap86], is used to calculate various values that are small increments of other values. For example, a hash table for $\mathrm{R}$ is assumed to occupy $F \cdot P_{r}$ pages in memory when overhead is included.

\begin{tabular}{|c|c|}
\hline Parameter & Definition \\
\hline $\begin{array}{l}\text { Mips } \\
P \\
I O \\
M \\
b \\
|\mathrm{R}| \\
k \\
\text { sel } \\
r \\
s \\
\text { compare }_{i} \\
\text { hash }_{i} \\
\text { bhash }_{i} \\
F\end{array}$ & $\begin{array}{l}\text { instruction execution rate } \\
\text { size of a disk page } \\
\text { time to read or write a disk page } \\
\text { number of memory buffer pages } \\
\text { B+ tree fanout } \\
\text { number of objects in } R \\
\text { sharing level } \\
\text { selectivity of the predicate on } R \\
\text { size of objects in } R \\
\text { size of objects in } S \\
\text { instructions to compare two values } \\
\text { instructions to hash a join attribute or PID } \\
\text { instructions to hash a value for bit filtering } \\
\text { fudge factor for hybrid-hash [Shap86] }\end{array}$ \\
\hline $\begin{array}{l}\text { compare } \\
\text { hash } \\
\text { bhash } \\
|S| \\
O_{r} \\
O_{s} \\
P_{r} \\
P_{s} \\
\text { move } \\
\text { move } \\
\text { swap, }_{r} \\
\text { swaps }_{\text {swa }}\end{array}$ & 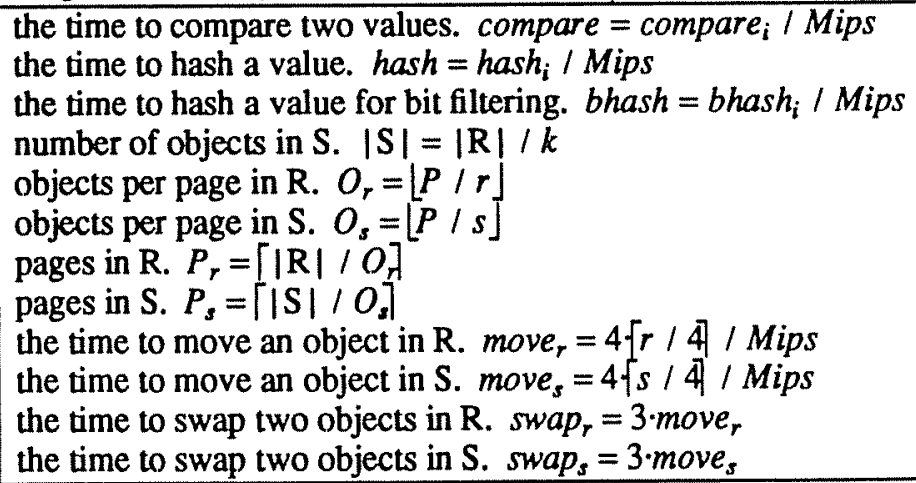 \\
\hline
\end{tabular}

Table 1: The Parameters Used in the Analysis 


\begin{tabular}{|l|l|}
\hline \multicolumn{2}{|l|}{ Defaults for Core Parameters } \\
\hline Mips & 10 (in millions of instructions per second) \\
IO & 20 milliseconds (fixed) \\
$P$ & 4096 bytes (fixed) \\
$M$ & 256 pages (or 1 Mbyte) \\
$b$ & 350 (fixed) \\
$|\mathrm{R}|$ & 100,000 objects \\
$k$ & 1 \\
sel & 0.01 \\
$r$ & 200 bytes \\
$s$ & 200 bytes \\
compare & 2 instructions (fixed) \\
hash $_{i}$ & 9 instructions (fixed) \\
bhash $_{i}$ & 3 instructions (fixed) \\
$F$ & 1.2 (fixed) \\
\hline
\end{tabular}

Table 2: The Defaults for the Core Parameters

It is important to note that there is only one $1 / O$ parameter; that is, we do not distinguish between sequential $\mathrm{I} / \mathrm{O}$ and random $\mathrm{I} / \mathrm{O}$. This is reasonable for small to medium-sized joins where the impact of sequential $\mathrm{L} / \mathrm{O}$ is likely to be negligible. For full joins, however, the impact of sequential $\mathrm{I} / \mathrm{O}$ can be significant if it is available. To gauge the impact of sequential I/O on full joins, we also obtained results for full joins with $P$ set to 32 Kbytes (i.e., tracksized disk transfers). We shall comment on those results later in the paper.

Also note that we do not consider CPU and I/O overlap in the analysis. We feel justified in doing this because all of the join algorithms would benefit from CPU and I/O overlap, and as a result, it should not significantly affect the relative performance of the algorithms. It is also questionable just how much CPU and I/O overlap is possible on a modern processor because of the large disparity between CPU and I/O speeds. For example, with the above parameter settings, almost 23,000 objects can be hashed in the time it takes to read a 32 Kbyte track containing only 160 objects. In such an environment, very little CPU and I/O overlap is likely to be possible, even if multi-track read-ahead is supported.

\subsection{Analysis of Large Joins}

In this section, full joins between $\mathrm{R}$ and $\mathrm{S}$ are analyzed. As mentioned earlier, index-nested-loops is not considered for such joins. Due to space limitations, we can only briefly touch on the analysis of the standard sort-merge and hybrid-hash algorithms here. For more details, the reader should consult [Shap86].

\subsubsection{Standard Sort-Merge}

Assuming enough memory is available, the cost of the two-pass sort-merge algorithm that we described earlier is [Shap86]: 


$$
\begin{aligned}
& \left(P_{r}+\ddot{P}_{s}\right) \cdot I O \\
& +|\mathrm{R}| \cdot \log _{2}|\mathrm{R}| \cdot\left(\text { compare }+ \text { swap }_{r}\right) \\
& +|\mathrm{S}| \cdot \log _{2}|\mathrm{~S}| \cdot\left(\text { compare }+ \text { swap }_{s}\right) \\
& +\left(P_{r}+P_{s}\right) \cdot 2 \cdot I O \\
& +(|\mathrm{R}|+|\mathrm{S}|) \cdot \text { compare } \\
& -\min \left(P_{s}+P_{r}, M-\sqrt{\left(P_{r}+P_{s}\right) / 2}\right) \cdot 2 \cdot I O
\end{aligned}
$$

read $R$ and $S$

manage the priority queues for $R$ manage the priority queues for $S$ read and write the output runs for $R$ and $S$ perform the final merge I/O savings if extra memory is available

In the final term, the value $\sqrt{\left(P_{r}+P_{s}\right) / 2}$ represents the minimal memory requirements of the algorithm. This is actually a more accurate estimate than the one presented in [Shap86]. To verify it, we have to show that approximately $\sqrt{\left(P_{r}+P_{s}\right) / 2}$ pages of memory are needed to simultaneously merge the sorted runs of $\mathrm{R}$ and $\mathrm{S}$ in memory, as required by the algorithm. This is verified by noting that each run of $\mathrm{R}$ will be on the average $2 \cdot M$ pages in length [Knut73]. Consequently, there will be approximately $P_{r} /(2 \cdot M)$ runs of $\mathrm{R}$, and approximately $P_{s} /(2 \cdot M)$ runs of $\mathrm{S}$. To simultaneously merge the sorted runs of $\mathrm{R}$ and $\mathrm{S}$ in memory, $M$ must therefore be large enough to satisfy:

$$
M \geq \frac{P_{r}}{2 \cdot M}+\frac{P_{s}}{2 \cdot M}
$$

This implies that:

$$
M \geq \sqrt{\left(P_{r}+P_{s}\right) / 2}
$$

Note that in the above analysis we have excluded the cost to write the result of the join to disk. This cost will be excluded in the analysis of all the algorithms. We have excluded it because the result of a join often forms the input of another database operation without ever being completely written to disk; moreover, this cost would be the same for all algorithms, anyway. Finally, note that the terms above group related costs in the algorithm, and their order does not coincide with the actual step-by-step execution of the algorithm. This same approach will be used throughout the analysis.

\subsubsection{Standard Hybrid-Hash}

To analyze the cost of the hybrid-hash algorithm, recall that it begins by dividing $\mathrm{R}$ into $B+1$ partitions $R_{0}, R_{1}, \ldots R_{B}$, and likewise for $\mathrm{S}$. The proper value of $B$ to make everything work is [Shap86]:

$$
B=\left\lceil\frac{F \cdot P_{r}-M}{M-1}\right\rceil
$$

When $\mathrm{R}$ is partitioned, $M-B$ pages of memory are allocated to build the hash table for $R_{0}$. The remainder of memory is allocated to serve as output buffers for $R_{1}, R_{2}, \ldots R_{B}$, with one page allocated per partition. $S$ is then partitioned in a similar fashion, except that $S_{0}$ is joined with $R_{0}$ as it is being partitioned. Letting $q$ equal the fraction of $\mathrm{R}$ represented by $R_{0}$ : 


$$
q=\frac{(M-B) / F}{P_{r}}
$$

the cost of the hybrid-hash algorithm is [Shap86]:

$\left(P_{r}+P_{s}\right) \cdot 1 O$

$+(|\mathrm{R}|+|\mathrm{S}|) \cdot$ hash

$+|\mathrm{R}| \cdot(1-q) \cdot$ move $_{r}$

$+|\mathrm{S}| \cdot(1-q) \cdot$ moves $_{\text {s }}$

$+P_{r} \cdot(1-q) \cdot 2 \cdot 10$

$+P_{s} \cdot(1-q) \cdot 2 \cdot 10$

$+(|\mathrm{R}|+|\mathrm{S}|) \cdot(1-q) \cdot h a s h$

$+|\mathrm{R}| \cdot$ mover $_{r}$

$+|\mathrm{S}| \cdot$ compare $\cdot F$ read $R$ and $S$

partition $R$ and $S$

move the objects in $R_{1}, R_{2}, \ldots R_{B}$ to their partition's output buffer move the objects in $S_{1}, S_{2}, \ldots S_{B}$ to their partition's output buffer read and write partitions $R_{1}, R_{2}, \ldots R_{B}$ read and write partitions $S_{1}, S_{2}, \ldots S_{B}$ hash the objects in each $R_{i}$ and probe with $S_{i}, 1 \leq i \leq B$ build the hash table for each $R_{i}, 0 \leq i \leq B$ probe the hash table for each object in $\mathrm{S}$ to find its match

Note that here we have assumed that $P_{r}<P_{s}$, otherwise the roles of $\mathrm{R}$ and $\mathrm{S}$ should be exchanged in the above analysis. As shown in [Shap86], the minimal memory requirements of the hybrid-hash algorithm are $\sqrt{F \cdot P_{r}}$ pages.

\subsubsection{Pointer-Based Nested-Loops}

In the pointer-based nested-loops algorithm, one page of memory is allocated to read $\mathrm{R}$ and the remainder are allocated to read S. Because of the way memory is allocated, and because $\mathrm{R}$ and $\mathrm{S}$ are relatively unclustered, this causes $S$ to be accessed in exactly the same manner as it would be in an unclustered index scan of $S$ with a buffer of size $M-1$. The I/O cost of an performing an unclustered index scan was derived in [Mack89]. Making use of the equations in that paper, the function $U_{s}(x, B)$, which counts the number of I/Os resulting from $x$ unclustered references to $S$ when a buffer of $B$ pages is used, is defined as:

let $q=\left(|S|-O_{s}\right) /|S|$ and let $p=1-q$

let $n=\max \left\{j\right.$ in $\{0,1, \ldots, x\}$ such that $\left.P_{s} \cdot\left(1-q^{j}\right) \leq B\right\}$

if $x \leq n$ then

$$
U_{s}(x, B)=P_{s} \cdot\left(1-q^{x}\right)
$$

else

$$
U_{s}(x, B)=P_{s} \cdot\left(1-q^{n}\right)+(x-n) \cdot P_{s} \cdot p \cdot q^{n}
$$

Using $U_{s}()$, the cost of accessing $S$ is therefore $U_{s}(|R|, M-1) \cdot I O$. We have defined $U_{s}()$ in this manner because it will be needed again later.

In addition to the cost of accessing $S$, there is also the cost to read $R$, and the CPU cost to check whether the page referenced by a given object in R is in memory, which we assume is done with hashing. The net cost of the 
pointer-based nested-loops algorithm is therefore:

$$
P_{r} \cdot I O+|\mathrm{R}| \cdot h a s h+U_{s}(|\mathrm{R}|, M-1) \cdot I O
$$

\subsubsection{Pointer-Based Sort-Merge}

The analysis of the pointer-based sort-merge algorithm is similar to the analysis of the standard sort-merge algorithm. The key difference here, of course, is that only $\mathrm{R}$ is sorted. Taking the analysis of the standard sortmerge algorithm and making the appropriate changes, the cost of the pointer-based sort-merge algorithm is:

$$
\begin{aligned}
& \left(P_{r}+P_{s}\right) \cdot I O \\
& +|\mathrm{R}| \cdot \log _{2}|\mathrm{R}| \cdot\left(\text { compare }+ \text { swap }_{r}\right) \\
& +P_{r} \cdot 2 \cdot 1 O \\
& -\min \left(P_{r}, M-\sqrt{P_{r} / 2}\right) \cdot 2 \cdot I O
\end{aligned}
$$

read $R$ and $S$

manage the priority queues for $R$ read and write the output runs for $R$ I/O savings if extra memory is available

Note that here the $\mathrm{I} / \mathrm{O}$ savings is a function of $\sqrt{P_{r} / 2}$, which corresponds to the minimal memory requirements of the algorithm. This is in contrast to the minimal memory requirements of the standard sort-merge algorithm, which as noted earlier are $\sqrt{\left(P_{r}+P_{s}\right) / 2}$ pages. Less memory is required here because only $\mathrm{R}$ is sorted.

\subsubsection{Pointer-Based Hybrid-Hash}

As one would expect, the analysis of the pointer-based hybrid-hash algorithm is similar to the analysis of the standard hybrid-hash algorithm. The main difference here is that only $\mathrm{R}$ is partitioned. Taking the analysis of the standard hybrid-hash algorithm and making the appropriate changes, the cost of the pointer-based hybrid-hash algorithm is:

$$
\begin{aligned}
& \left(P_{r}+P_{s}\right) \cdot I O \\
& +|\mathrm{R}| \cdot \text { hash } \\
& +|\mathrm{R}| \cdot(1-q) \cdot \text { move }_{r} \\
& +P_{r} \cdot(1-q) \cdot 2 \cdot I O \\
& +|\mathrm{R}| \cdot(1-q) \cdot \text { hash } \\
& +|\mathrm{R}| \cdot \text { move }_{r}
\end{aligned}
$$

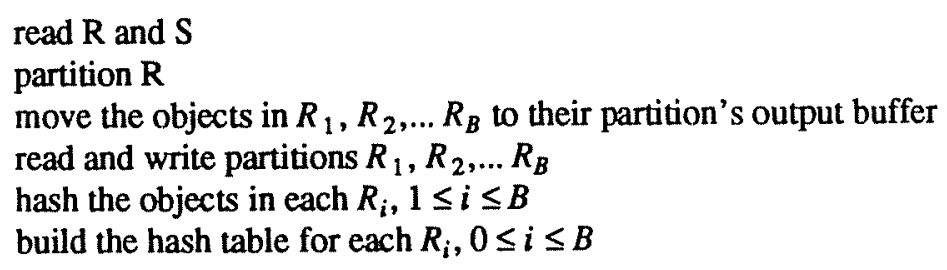

To determine the minimal memory requirements of the pointer-based hybrid-hash algorithm, it suffices to note that in [Shap86] the minimal memory requirements of the standard hybrid-hash algorithm were shown to equal $\sqrt{F \cdot P}$ pages, where $P$ is the number of pages in the inner set. The minimal memory requirements were not affected by the size of the outer set. From this it follows that the minimal memory requirements of the pointer-based hybrid-hash algorithm are $\sqrt{F \cdot P_{r}}$ pages, since $\mathrm{R}$ always plays the role of the inner set here. 


\subsection{Analysis of Small to Medium-Sized Joins}

In this section, we analyze small to medium-sized joins, where the result of a selection on $R$ is joined to $S$. As mentioned earlier, the analysis will assume that $B+$ tree indexes exist on both $R$ and $S$. The index on $R$ will be used to evaluate the selection predicate, while the index on $\mathrm{S}$, which is assumed to be a unique primary-key index, will be used by the index-nested-loops algorithm to avoid a file scan of S.

The only index clustering combination that will be analyzed in this section is the combination where the index on $R$ is a clustered index and the index on $S$ is an unclustered index. We will denote this combination as clustered/unclustered, and similarly for the other combinations. The clustered/unclustered index combination has been chosen here because it is perhaps the most difficult to analyze. Note that we will still present results for the unclustered/clustered index combination, as well comment on how the results for the two remaining index combinations compare to the results that are presented. In addition, the analysis for the unclustered/clustered index combination is presented in the Appendix. Using that analysis and the analysis of this section, the remaining index combinations are straightforward to analyze.

Another thing to note is that, throughout this section, we will ignore the small amount of memory space that is required to read the indexes on R and S. A small, two-page MRU buffer group [Chou85] would be sufficient for reading either of these indexes in the examples that are analyzed, and accounting for that little space would not change the results in any significant way. In addition, we will assume that the selectivity of the predicate on $R$ is such that $R^{\prime}$, which is defined as the result of the selection on $R$, fits completely in memory. In contrast to the indexes on $\mathrm{R}$ and $\mathrm{S}$, we will account for the memory space used by $\mathrm{R}^{\prime}$.

Finally, to simplify the equations of this section, we let $\left|R^{\prime}\right|$ denote the cardinality of $\mathrm{R}^{\prime}$ and $P_{r}{ }^{\prime}$ denote the number of pages in $\mathrm{R}^{\prime}$, that is, we let $\left|\mathrm{R}^{\prime}\right|=\operatorname{sel} \cdot|\mathrm{R}|$ and $P_{r}{ }^{\prime}=\operatorname{sel} \cdot P_{r}$.

\subsubsection{Standard Index-Nested-Loops}

The index-nested-loops algorithm begins by using the $B+$ tree index on $R$ to obtain $R^{\prime}$. This is done by descending the index to a leaf, and then scanning across the leaves to obtain the objects in $\mathrm{R}$ that satisfy the selection predicate. In all the examples that are analyzed, the height of the index on $\mathrm{R}$ is equal to 2 , and similarly for $\mathrm{S}$. The cost of reading the index on $\mathbf{R}$ is therefore:

$$
\begin{array}{ll}
2 \cdot I O & \text { IO cost to descend the index } \\
+2 \cdot \log _{2} b \cdot \text { compare } & \text { CPU cost to descend the index } \\
+\left\lceil\left|R^{\prime}\right| \mid b-1\right\rceil \cdot I O & \text { scan across the leaves of the index }
\end{array}
$$

$\mathrm{R}^{\prime}$ is then read into memory using the index and sorted by foreign key (i.e., by join attribute). This costs: 


$$
\begin{array}{ll}
P_{r}^{\prime} \cdot I O^{\prime} & \text { cost to read } R^{\prime} \\
+\left|R^{\prime}\right| \cdot \text { move }_{r} & \text { extract } R^{\prime} \text { from } R \text { in memory before sorting } \\
+\left|R^{\prime}\right| \cdot \log _{2}\left|R^{\prime}\right| \cdot\left(\text { compare }+ \text { swap }_{r}\right) & \text { cost to sort } R^{\prime}
\end{array}
$$

After $R^{\prime}$ has been sorted, the foreign key in each object of $R^{\prime}$ is used to probe the index on $S$. Assuming that the height of the index is 2 , the $\mathrm{I} / \mathrm{O}$ cost to probe the index consists of the cost to read the root page of the index plus the cost of reading whatever leaf pages of the index are accessed by the index probes. Because $\mathrm{R}^{\prime}$ is sorted by foreign key, the leaf pages will be accessed in ascending key order, and consequently a simple two-page MRU buffer group will ensure that no leaf page is read more than once. All that remains, therefore, is to determine the number of leaf pages that are accessed by the index probes.

To determine the number of leaf pages that are accessed by the index probes, we first consider the probability that a particular leaf page $L_{i}$ is not accessed. Since $\mathrm{R}$ and $\mathrm{S}$ are relatively unclustered, we can assume that any particular leaf page of the index is just as likely to be accessed as any other leaf page. Therefore, the probability that a leaf page $L_{i}$ is not accessed is equal to the probability of choosing a subset of $\left|R^{\prime}\right|$ objects from $R$ such that the chosen subset contains no object with a foreign key in $L_{i}$. Since each leaf page contains $b$ keys, and since there are $k$ objects in $\mathrm{R}$ that share each key value, the probability that $L_{i}$ is not accessed is:

$$
\operatorname{Prob}\left(L_{i} \text { is not accessed }\right)=\frac{\left(\begin{array}{c}
|\mathrm{R}|-b \cdot k \\
\left|\mathrm{R}^{\prime}\right|
\end{array}\right]}{\left[\begin{array}{l}
|\mathrm{R}| \\
\left|\mathrm{R}^{\prime}\right|
\end{array}\right]}
$$

Since there are a total of $\lceil|S| / B]$ leaf pages, the expected number of leaf pages that are accessed by the index probes is therefore:

$$
\text { expected leaf pages accessed }=[|\mathrm{S}| / b] \cdot\left[1-\frac{\left(\begin{array}{c}
|\mathrm{R}|-b \cdot k \\
\left|\mathrm{R}^{\prime}\right|
\end{array}\right]}{\left[\begin{array}{l}
|\mathrm{R}| \\
\left|\mathrm{R}^{\prime}\right|
\end{array}\right]}\right]
$$

This same quantity was derived in another context [Yao77]. If we let $Y($ ) denote the so-called Yao function:

$$
Y(u, v, w)=1-\frac{\left(\begin{array}{c}
u-v \\
w
\end{array}\right)}{\left[\begin{array}{l}
u \\
w
\end{array}\right]}
$$

then the total cost to probe the index on $S$ is:

10

$+2 \cdot\left|\mathrm{R}^{\prime}\right| \cdot \log _{2} b \cdot$ compare

$+[|\mathrm{S}| / b] \cdot Y\left(|\mathrm{R}|, b \cdot k,\left|\mathrm{R}^{\prime}\right|\right) \cdot I O$ read the root page

probe the index for each object in $\mathrm{R}^{\prime}$

access the leaf pages 
The final cost that needs to be included is the cost to read $S$. Here, we assume that $S$ is read using all of the available memory that is not allocated to hold $R^{\prime}$. In effect, this means that $S$ ends up being read in the same manner as in the pointer-based nested-loops algorithm, except that here index pointers take the place of object pointers from $\mathrm{R}$; in addition, $\mathrm{S}$ is referenced $\left|\mathrm{R}^{\prime}\right|$ times now and there are only $M-P_{r}^{\prime}$ memory pages available to read $\mathrm{S}$. Based on these observations and using the $\mathrm{H} / \mathrm{O}$ function $U_{s}($ ) that was defined earlier, the cost to read $\mathrm{S}$ is simply:

$$
\begin{array}{ll}
\left|\mathrm{R}^{\prime}\right| \cdot h a s h & \text { check memory for each index reference to } \mathrm{S} \\
+U_{s}\left(\left|\mathrm{R}^{\prime}\right|, M-P_{r}\right) \cdot I O & \text { access } \mathrm{S}
\end{array}
$$

Before going on to the analysis of the sort-merge algorithm, it is important to point out that we also analyzed the normal index-nested-loop algorithm where $\mathrm{R}^{\prime}$ is not sorted. In all of the examples that we will consider, it performed considerably worse than the algorithm that we have described due to the cost of also accessing the leaf pages of the index on $S$ in a random fashion.

\subsubsection{Standard Sort-Merge}

The sort-merge algorithm begins by reading $R^{\prime}$ into memory and sorting it, just as in the index-nested-loops algorithm. In addition, each object in $\mathrm{R}^{\prime}$ is also hashed to turn on a bit in the bit filter. Here we shall assume that only one hash function is used for bit filtering. In the examples that are analyzed, a simple page-sized bit filter using one hash function turns out to be sufficient to filter out virtually all of the non-participating objects in S. (Details on how to calculate the optimal number of hash functions to use, and what the effectiveness of the resulting bit filter is, can be found in [Seve76].)

After $\mathrm{R}^{\prime}$ has been sorted, $\mathrm{S}$ is read into memory, filtered, and the resulting objects are sorted. As mentioned, we are assuming that virtually all of the non-participating objects in $S$ are screened out by the bit filter. Consequently, only the objects in $S$ that join with $\mathrm{R}^{\prime}$ are sorted. Let $S^{\prime}$ denote these objects, and let $\left|S^{\prime}\right|$ and $P_{s}^{\prime}$ denote the cardinality and the number of pages in $S^{\prime}$, respectively. To determine $\left|S^{\prime}\right|$, the same sort of analysis that was used to determine the number of index leaf pages accessed by the index-nested-loops algorithm can be applied. The analysis proceeds by considering the probability that no object in $\mathrm{R}^{\prime}$ joins with a given object in $S$. Since each object in $\mathrm{S}$ joins with $k$ objects in $\mathrm{R}$, a similar application of the previous analysis yields:

$$
\left|\mathrm{S}^{\prime}\right|=|\mathrm{S}| \cdot Y\left(|\mathrm{R}|, k,\left|\mathrm{R}^{\prime}\right|\right)
$$

The corresponding value for $P_{s}{ }^{\prime}$ is simply $P_{s}^{\prime}=\left|\mathrm{S}^{\prime}\right| / O_{s}$. Based on the analysis of the standard sort-merge algorithm for full joins, and based on the analysis of the index-nested-loops algorithm on small to medium-sized joins, the cost of the sort-merge algorithm is: 
$2 \cdot I O+2 \cdot \log _{2} b \cdot$ compare

$+\left[\left|R^{\prime}\right| / b-1\right] \cdot 10$

$+\left(P_{r}{ }^{\prime}+P_{s}\right) \cdot I O$

$+\left(\left|\mathbf{R}^{\prime}\right|+|\mathbf{S}|\right) \cdot$ bhash

$+\left|R^{\prime}\right| \cdot$ move $_{r}$

$+\left|S^{\prime}\right| \cdot$ moves $_{s}$

$+\left|\mathbf{R}^{\prime}\right| \cdot \log _{2}\left|\mathbf{R}^{\prime}\right| \cdot\left(\right.$ compare + swap $\left._{r}\right)$

$+\left|S^{\prime}\right| \cdot \log _{2}\left|S^{\prime}\right| \cdot\left(\right.$ compare + swap $\left._{s}\right)$

$+\left(P_{r}{ }^{\prime}+P_{s}\right) \cdot 2 \cdot I O$

$+\left(\left|R^{\prime}\right|+\left|S^{\prime}\right|\right) \cdot$ compare

$-\min \left(P_{s}^{\prime}+P_{r}^{\prime}, M-\sqrt{\left(P_{r}^{\prime}+P_{s}^{\prime}\right) / 2}\right) \cdot 2 \cdot 1 O$ descend the index on $R$

scan across the leaves of the index on $R$ read $R^{\prime}$ and $S$

bit filter $R^{\prime}$ and $S$

extract $R^{\prime}$ from $R$ in memory before sorting

extract $S^{\prime}$ from $S$ in memory before sorting

manage the priority queues for $R^{\prime}$

manage the priority queues for $S^{\prime}$

read and write the output runs for $R^{\prime}$ and $S^{\prime}$

perform the final merge

I/O savings if extra memory is available

\subsubsection{Standard Hybrid-Hash}

The analysis of the hybrid-hash algorithm follows from the analysis that was used earlier on full joins and from the analysis of the standard sort-merge algorithm on small to medium-sized joins. With $S^{\prime}$ defined the same way it was in the analysis of the sort-merge algorithm, and with our assumption that $R^{\prime}$ fits in memory, the cost of the hybrid-hash algorithm is:

$2 \cdot 1 O+2 \cdot \log _{2} b \cdot$ compare

$+\left[\left|\mathrm{R}^{\prime}\right| / b-1\right\rceil \cdot I O$

$+\left(P_{r}^{\prime}+P_{s}\right) \cdot I O$

$+\left(\left|\mathbf{R}^{\prime}\right|+|\mathrm{S}|\right) \cdot$ bhash

$+\left(\left|R^{\prime}\right|+\left|S^{\prime}\right|\right) \cdot$ hash

$+\left|\mathbf{R}^{\prime}\right| \cdot$ mover $_{r}$

$+\left|\mathrm{S}^{\prime}\right| \cdot$ compare $\cdot F$ descend the index on $R$

scan across the leaves of the index on $R$

read $R^{\prime}$ and $S$

bit filter $R^{\prime}$ and $S$

partition $R^{\prime}$ and $S^{\prime}$

build the hash table for $\mathrm{R}^{\prime}$

probe the hash table for each object in $S^{\prime}$ to find its match

\subsubsection{Pointer-Based Nested-Loops}

The analysis of the pointer-based nested-loops algorithm follows almost directly from the analysis that was used earlier on full joins. Taking that analysis and making some straightforward changes, the cost of the pointerbased nested-loops algorithm is:

$$
\begin{aligned}
& 2 \cdot I O+2 \cdot \log _{2} b \cdot c o m p a r e \\
& +\left[\left|\mathrm{R}^{\prime}\right| / b-1\right] \cdot I O \\
& +P_{r}^{\prime} \cdot I O \\
& +\left|\mathrm{R}^{\prime}\right| \cdot \text { hash } \\
& +U_{s}\left(\left|\mathrm{R}^{\prime}\right|, M-1\right) \cdot I O
\end{aligned}
$$

descend the index on $\mathbf{R}$

scan across the leaves of the index on $R$ read $\mathrm{R}^{\prime}$ check memory for each pointer reference in $\mathrm{R}^{\prime}$ access $\mathbf{S}$

\subsubsection{Pointer-Based Sort-Merge}

In order to analyze the pointer-based sort-merge algorithm, we need to estimate how many pages in $\mathrm{S}$ participate in the join. Let $P_{s}{ }^{\prime}$ denote number of pages in $\mathrm{S}$ that participate in the join. (Note that $P_{s}^{\prime}$ as defined here is not the same as the $P_{s}^{\prime}$ that was defined in the standard algorithms.) To determine $P_{s}^{\prime}$, the analysis that was used to 
determine the number of index leaf pages accessed by the index-nested-loops algorithm can be applied once again. The analysis proceeds by considering the probability that no object in $R^{\prime}$ joins with a given page in $S$. Since each page in $\mathrm{S}$ joins with $k \cdot O_{s}$ objects in $\mathrm{R}$, a similar application of the previous analysis yields:

$$
P_{s}^{\prime}=P_{s} \cdot Y\left(|\mathrm{R}|, k \cdot O_{s},\left|\mathrm{R}^{\prime}\right|\right)
$$

With the exception of $P_{s}^{\prime}$, the rest of the analysis is straightforward and follows from the analysis that was used earlier on full joins. Under our assumption that $R^{\prime}$ fits in memory, the cost of the pointer-based sort-merge algorithm is:

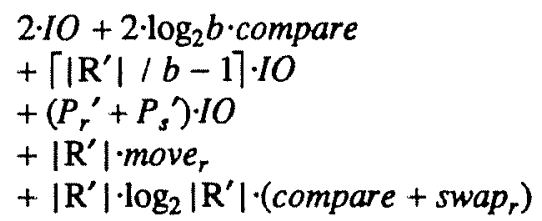

descend the index on $\mathbf{R}$ scan across the leaves of the index on $R$ read $R^{\prime}$ and $S^{\prime}$ extract $R^{\prime}$ from $R$ in memory before sorting manage the priority queues for $\mathrm{R}^{\prime}$

\subsubsection{Pointer-Based Hybrid-Hash}

The analysis of the hybrid-hash algorithm follows directly from the analysis that was used earlier on full joins and also from the analysis of the pointer-based sort-merge algorithm on small to medium-sized joins. With $P_{s}{ }^{\prime}$ defined as in the analysis of the pointer-based sort-merge algorithm, and with our assumption that $\mathrm{R}^{\prime}$ fits in memory, the cost of the pointer-based hybrid-hash algorithm is:

$\begin{array}{ll}2 \cdot I O+2 \cdot \log _{2} b \cdot \text { compare } & \text { descend the index on } \mathrm{R} \\ +\left\lceil\left|\mathrm{R}^{\prime}\right| \cdot b-1\right\rceil \cdot I O & \text { scan across the leaves of the index on } \mathrm{R} \\ +\left(P_{r}^{\prime}+P_{s}^{\prime}\right) \cdot I O & \text { read } \mathrm{R}^{\prime} \text { and } \mathrm{S}^{\prime} \\ +\left|\mathrm{R}^{\prime}\right| \cdot \text { hash } & \text { hash } \mathrm{R}^{\prime} \\ +\left|\mathrm{R}^{\prime}\right| \cdot \text { move }_{r} & \text { build the hash table for } \mathrm{R}^{\prime}\end{array}$

\subsection{Performance Results for Large Joins}

The results for full joins between $R$ and $S$ are presented in Figure 3. The graphs were obtained by using the equations from the analysis to compute the total time in seconds to run each join algorithm for a particular memory size. The size of memory was increased in $1 / 2 \mathrm{Mbyte}$ increments and ranged from the minimal size required to execute all the join algorithms, which was approximately $1 / 4$ Mbytes, all the way up to 10 Mbytes. In all of the graphs, the lines for the standard, non-pointer-based algorithms are labeled wo/ptr for "without pointer", while the lines for the pointer-based algorithms are labeled w/ptr for "with pointer".

Four graphs are shown in Figure 3. Graph A shows the performance of the join algorithms when $\mathrm{R}$ and $\mathrm{S}$ are the same size (in pages). In that graph, both $R$ and $S$ consist of 100,000 objects with 200 bytes per object. As shown, the pointer-based algorithms can provide significant performance gains in this situation. Compared to the 
standard hybrid-hash algorithm, the pointer-based hybrid-hash algorithm reduces the join time by approximately $30 \%$ over the whole range of memory sizes considered. This, of course, is because $S$ is not partitioned in the pointer-based hybrid-hash algorithm. A similar relationship is seen between the standard sort-merge algorithm and the pointer-based sort-merge algorithm.

One of the interesting things to notice about Graph A is just how poorly the pointer-based nested-loops algorithm performs. As mentioned earlier, this is because it does not try to optimize its disk reads of $S$ like the other pointer-based algorithms. These results demonstrate that, even with pointers, something more intelligent than a nested-loops approach (or naive pointer traversal) is often needed for high performance.

Graph B shows the performance of the join algorithms when $R$ is ten times the size of $S$. In this case, the pointer-based hybrid-hash algorithm takes three times longer to execute than the standard hybrid-hash algorithm when the size of memory is 2.5 Mbytes, which is the amount of memory that would presumably be allocated by a query optimizer to execute the standard hybrid-hash algorithm. This large difference in performance is due to the fact that the pointer-hased hybrid-hash algorithm always partitions $\mathrm{R}$ first, which in this case is a poor choice because $\mathrm{R}$ is so much bigger than $\mathrm{S}$. Note also that the pointer-based nested-loops algorithm performs as well as or better than the standard hybrid-hash algorithm when the size of memory is 2 Mbytes or more. This is because all of $S$ actually fits in memory in that case.

Finally, the graphs in the lower half of Figure 3 further demonstrate how the relative performance of the pointer-based algorithms depends on the size ratio of $R$ and $S$. If $R$ is roughly the same size as $S$ or smaller, then the pointer-based algorithms always perform better. Otherwise, the standard algorithms perform better, unless there is enough memory available to hold $S$, in which case the pointer-based nested-loops algorithm performs as well as the standard hybrid-hash algorithm.

Graph $\mathrm{C}$ shows the performance of the algorithms when $\mathrm{R}$ is one fifth the size of $\mathrm{S}$. The graph has roughly the same shape as Graph A, but in this case, the pointer-based hybrid-hash and sort-merge algorithms outperform their standard counterparts by up to 55\%. This is because the relative cost to partition or sort $S$ in the standard algorithms is larger in this case due to its increased size.

Graph D shows the performance of the algorithms when $\mathrm{R}$ is twice the size of $\mathrm{S}$. This is perhaps the most interesting of all the graphs because of the way the lines for the pointer-based and standard hybrid-hash algorithms cross each other. As shown, the pointer-based hybrid-hash algorithm is better when there are 6 Mbytes of memory or less, but beyond that, the standard hybrid-hash algorithm is better. The reason for the crossover is because the pointer-based hybrid-hash algorithm always chooses $R$ as its inner set, which is a poor choice in this case because $R$ is larger than $\mathrm{S}$. However, even with $\mathrm{R}$ as the inner set, the pointer-based hybrid-hash algorithm performs better ini- 


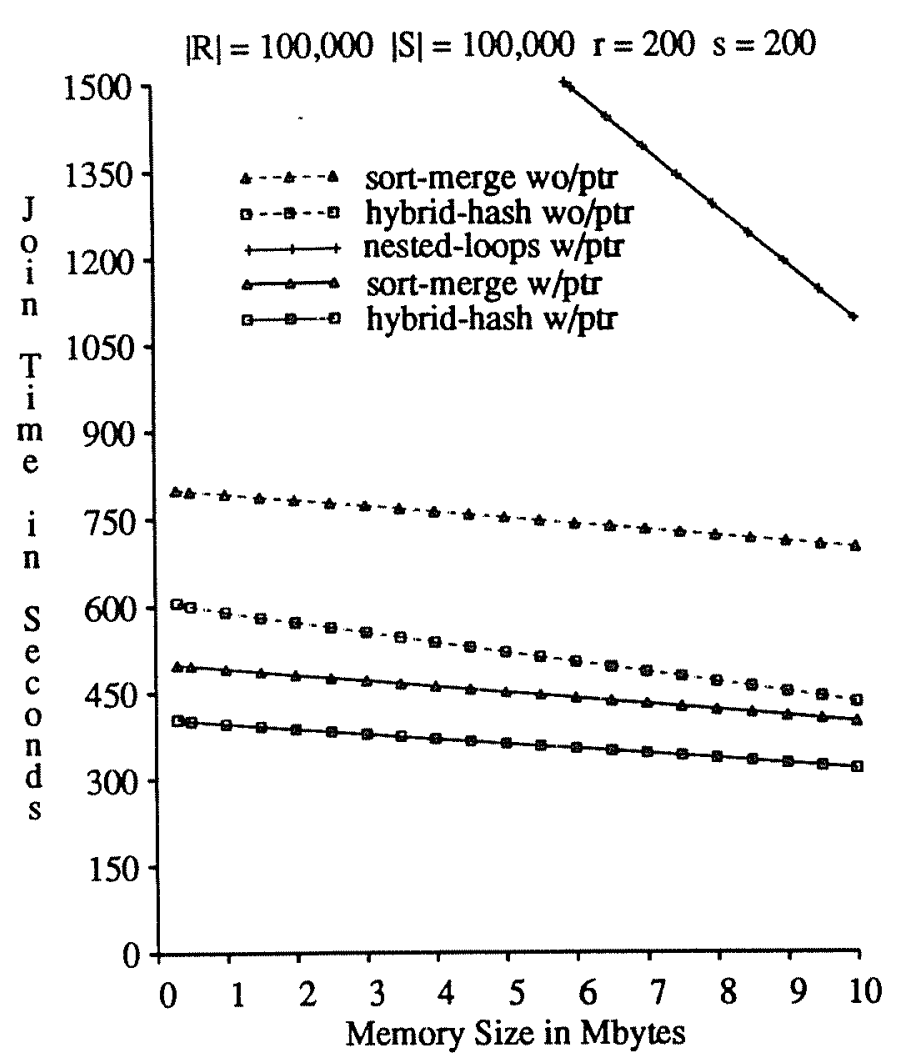

(A)

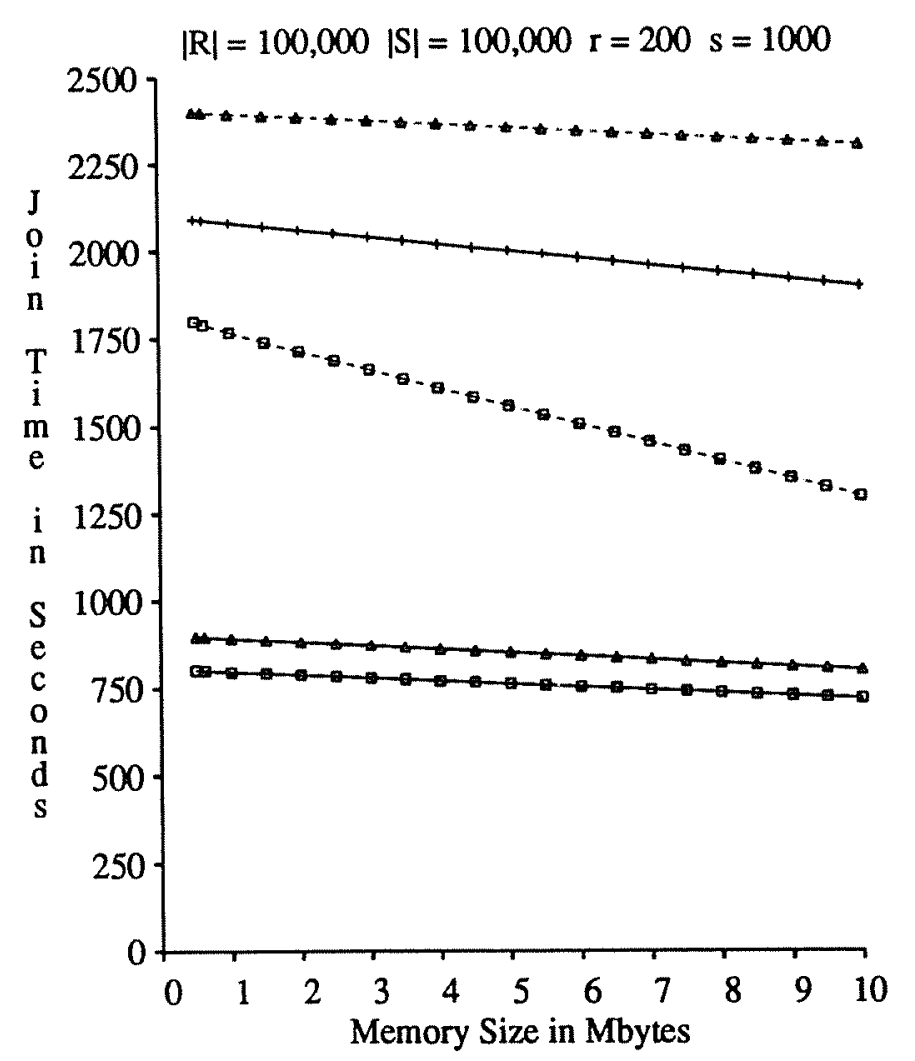

(C)

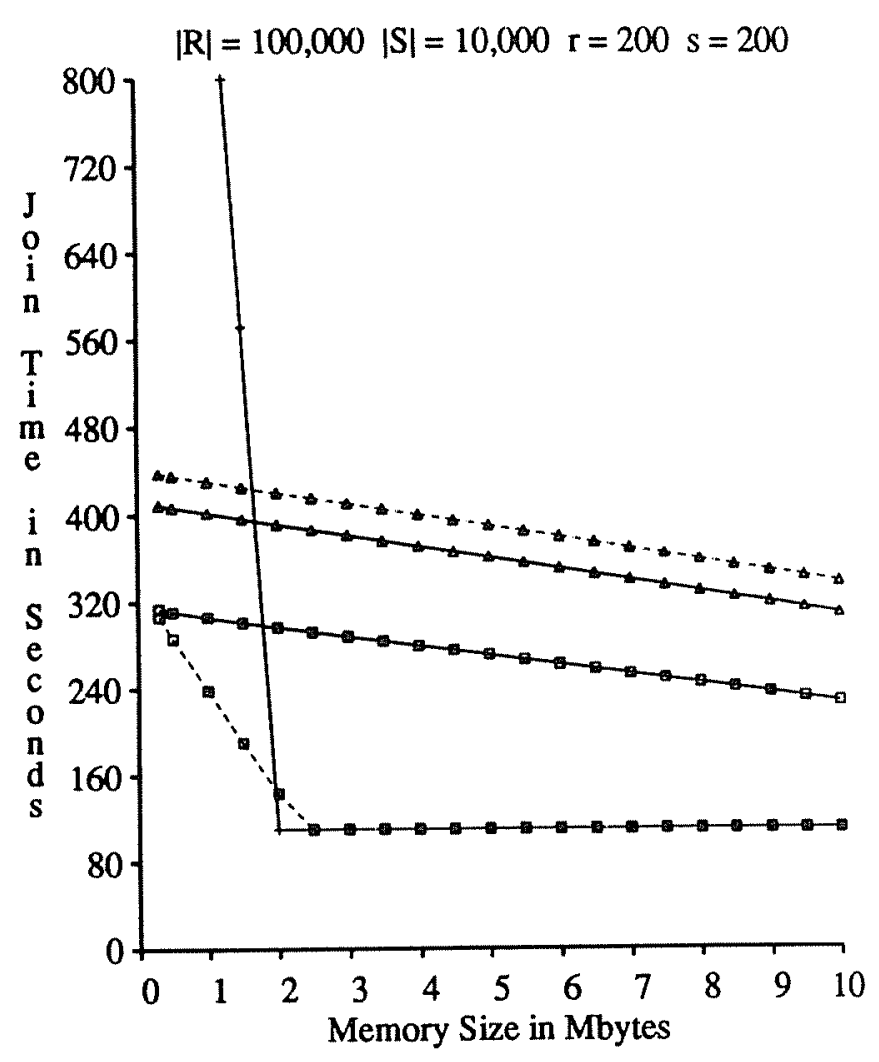

(B)

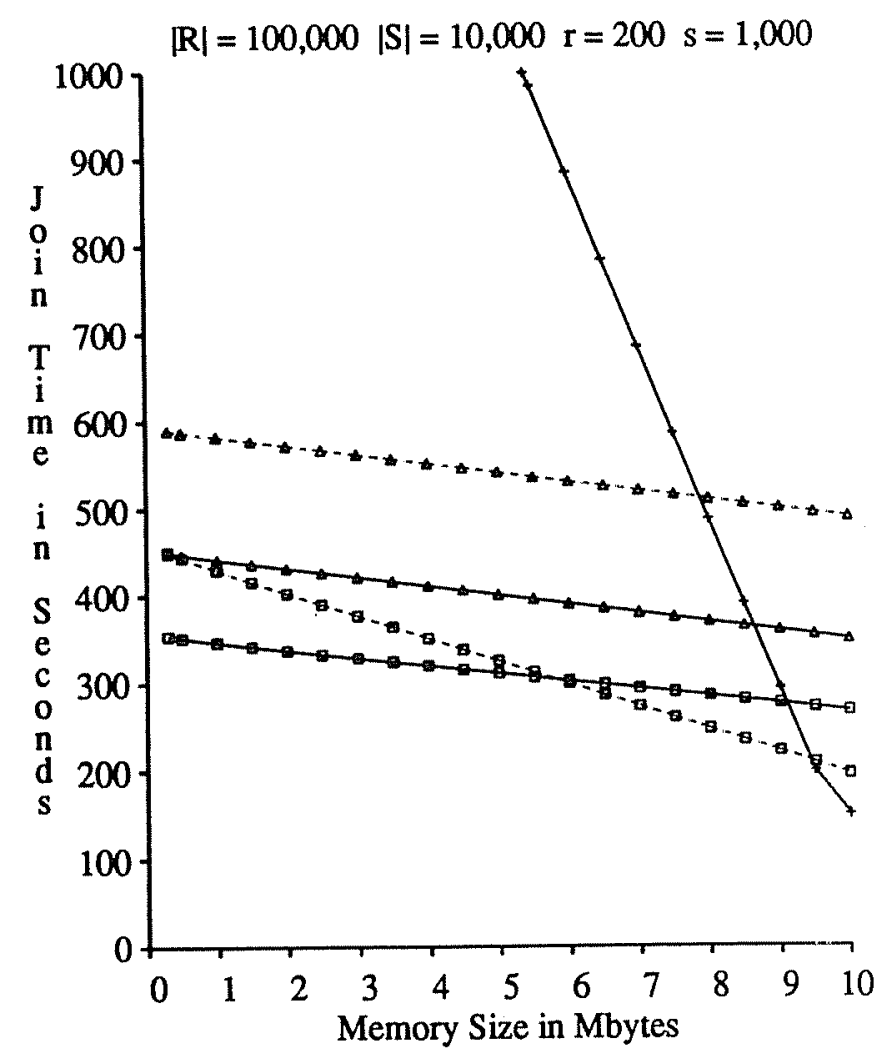

(D)

Figure 3: Results for Full Joins 
tially because it-does not have to partition $S$. With 6 Mbytes or more of memory, though, enough of $S$ fits in memory so that the savings from not partitioning $S$ are insufficient to offset the added costs of using $R$ as the inner set; at that point, the standard hybrid-hash algorithm starts to perform better.

As noted earlier, we also obtained full-join results with the size of the disk transfer unit, $P$, set to 32 Kbytes in order to gauge the impact of sequential $\mathrm{H} / \mathrm{O}$. When this was done, the join times decreased by about $85 \%$ for both of the hash-based algorithms and by about $65 \%$ for both of the sort-based algorithms (in all of the graphs). The join time for the pointer-based nested-loops algorithm decreased by about $10 \%$ except when $S$ fit in memory, in which case its join time was again about the same as that of the standard hybrid-hash algorithm. These results demonstrate that the pointer-based algorithms benefit from sequential LO as much as their standard counterparts do. The hashbased algorithms benefit more than the sort-based algorithms because they are more $1 / O$ bound. The pointer-based nested-loops algorithms benefits the least from sequential $\mathrm{I} / \mathrm{O}$ because large disk transfers do not significantly lower its cost to read $\mathrm{S}$, which is accessed in a random fashion.

Finally, it is important to note that, on the joins in which they perform the best, the CPU times of the pointerbased join algorithms are significantly less than that of their standard counterparts. (This is not always true on the joins in which they perform worse.) Therefore, in a CPU bound system, the pointer-based algorithms could improve performance by even larger margins than those suggested by Graphs A and C. Table 3 gives a rough feeling for how widely the CPU times differ among the join algorithms. That table shows the CPU time of each algorithm for the join in Graph $C$ with 1,5, and 10 Mbytes of memory.

\subsection{Performance Results for Medium-Sized Joins}

The results for medium-sized joins are presented in Figure 4. In all of these graphs, the size of $R$ was kept fixed at 100,000 objects, and the selectivity of the predicate on $R$ was kept fixed at 0.01 . Consequently, each graph represents the join of 1,000 objects in $R$ with $S$. As shown, the size of memory was increased in 1/8 Mbyte increments and ranged from the minimal size required to hold the selection on $\mathrm{R}$ in memory, which was approximately $1 / 4$ Mbytes, all the way up to 2 Mbytes.

CPU Time in Seconds

\begin{tabular}{|l|c|c|c|}
\hline memory size & 1 Mbytes & 5 Mbytes & 10 Mbytes \\
\hline sort-merge wo/ptr & 598.65 & 598.65 & 598.65 \\
\hline hybrid-hash wo/ptr & 13.91 & 11.79 & 9.19 \\
\hline nested-loops w/ptr & 0.90 & 0.90 & 0.90 \\
\hline sort-merge w/ptr & 100.00 & 100.00 & 100.00 \\
\hline hybrid-hash w/ptr & 4.10 & 3.74 & 3.29 \\
\hline
\end{tabular}

Table 3: CPU Times for Graph C 
The graphs in the upper half of Figure 4 are for the clustered/unclustered index combination, where the index on $\mathrm{R}$ is a clustered index and the index on $\mathrm{S}$ is an unclustered index. These graphs represent the situation where the pointer-based algorithms perform their best in relation to the index-nested-loops algorithm, since the relative cost of accessing $S$ via its index is high in this case.

Graph $E$ shows the performance of the join algorithms when $R$ is the same size as $S$. As shown, compared to the index-nested-loops algorithm, the pointer-based algorithms reduce the join time by approximately $30 \%$ over the whole range of memory sizes considered. This, of course, is because the index on $\mathrm{S}$ is not read by the pointer-based algorithms. Note that the standard sort-merge and hybrid-hash algorithms perform poorly here because, unlike their pointer-based counterparts, they read all of S, even though only a small fraction of S actually participates in the join.

Graph $\mathrm{F}$ shows the performance of the join algorithms when $\mathrm{R}$ is ten times the size of $\mathrm{S}$. In this case, the fraction of $S$ that participates in the join is large enough so that both the index-nested-loops algorithm and the pointerbased nested-loops algorithm perform poorly in relation to the other join algorithms. This is because neither of these algorithms try to optimize their disk reads of S. Compared to the standard hybrid-hash algorithm, the pointerbased hybrid-hash algorithm reduces the join time by approximately $15 \%$ over the whole range of memory sizes considered. A similar relationship is seen between the standard sort-merge algorithm and the pointer-based sortmerge algorithm.

The graphs in the lower half of Figure 4 are for the same cases as in the top half of the figure, but with the unclustered/clustered index combination. It should be clear that these graphs represent the situation where the pointer-based algorithms perform their worst in relation to the index-nested-loops algorithm. This is because the relative cost of accessing $S$ via its index is low in this case. As shown, the pointer-based algorithms perform approximately $15 \%$ better than the index-nested-loops algorithm in Graph $\mathrm{G}$ and approximately $5 \%$ better in Graph H.

The graphs for the clustered/clustered and unclustered/unclustered index combinations have not been presented here. Although those graphs do not follow quite the same patterns as those in Figure 4, similar benefits and tradeoffs were observed; the pointer-based algorithms always outperformed their standard counterparts.

Before discussing the results for small joins, it is important to emphasize that the CPU times of the pointerbased algorithms are significantly less than their standard counterparts on all the medium-sized joins. This holds true for the small joins as well. For example, in Graph E the CPU time for the index-nested-loops algorithm is $624.22 \mathrm{msec}$, whereas it is only $0.90 \mathrm{msec}$ for the pointer-based nested-loops algorithm. In a CPU bound system, such a large a difference in CPU times could have a significant impact on performance. 


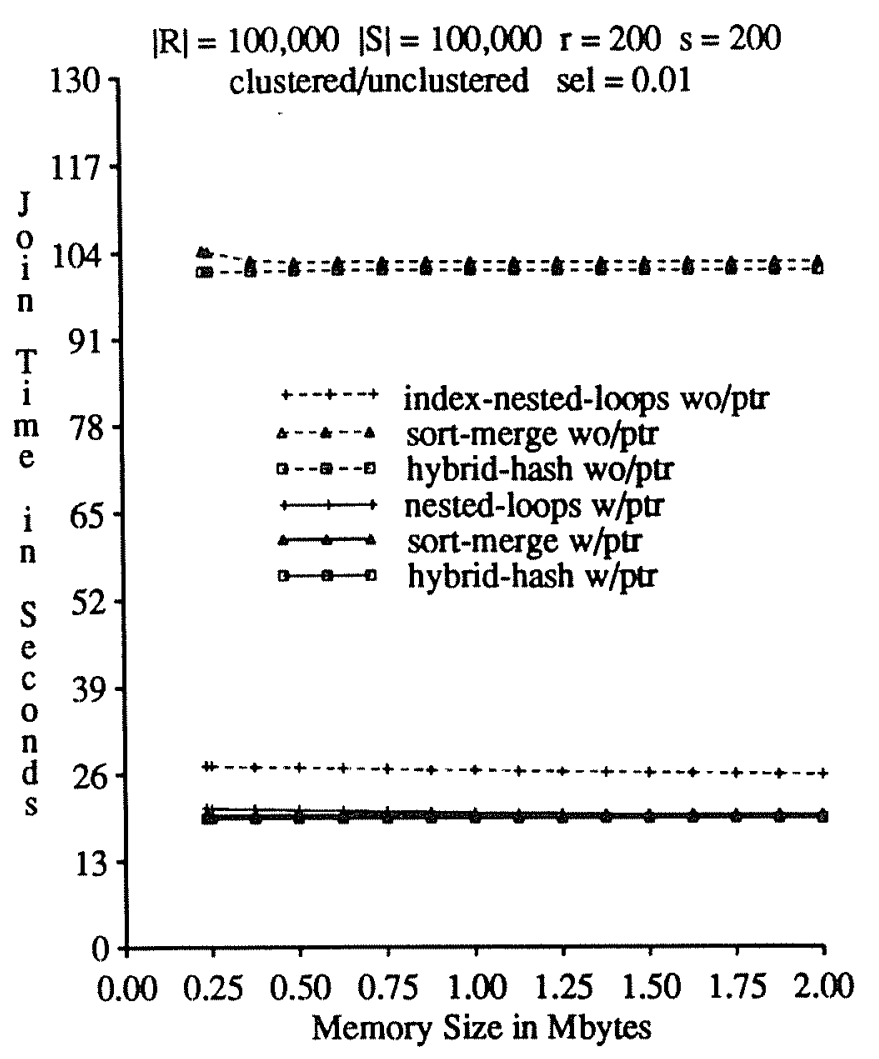

(E)

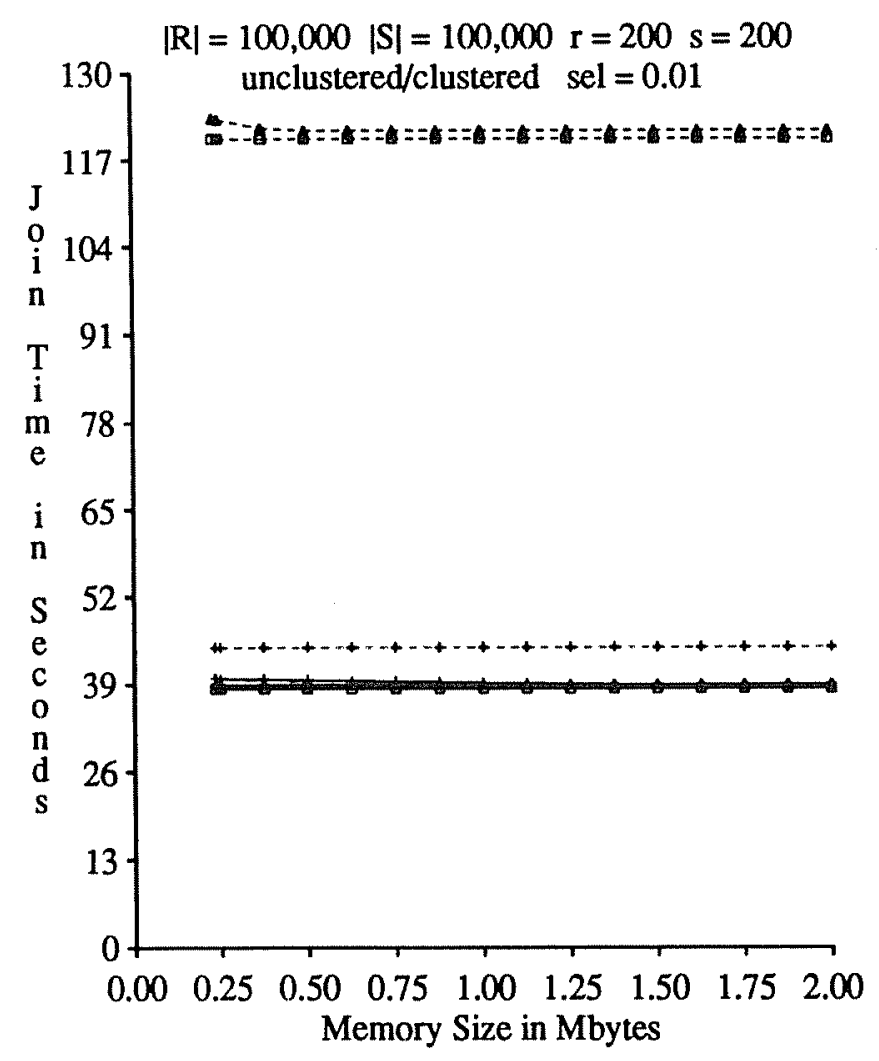

(G)

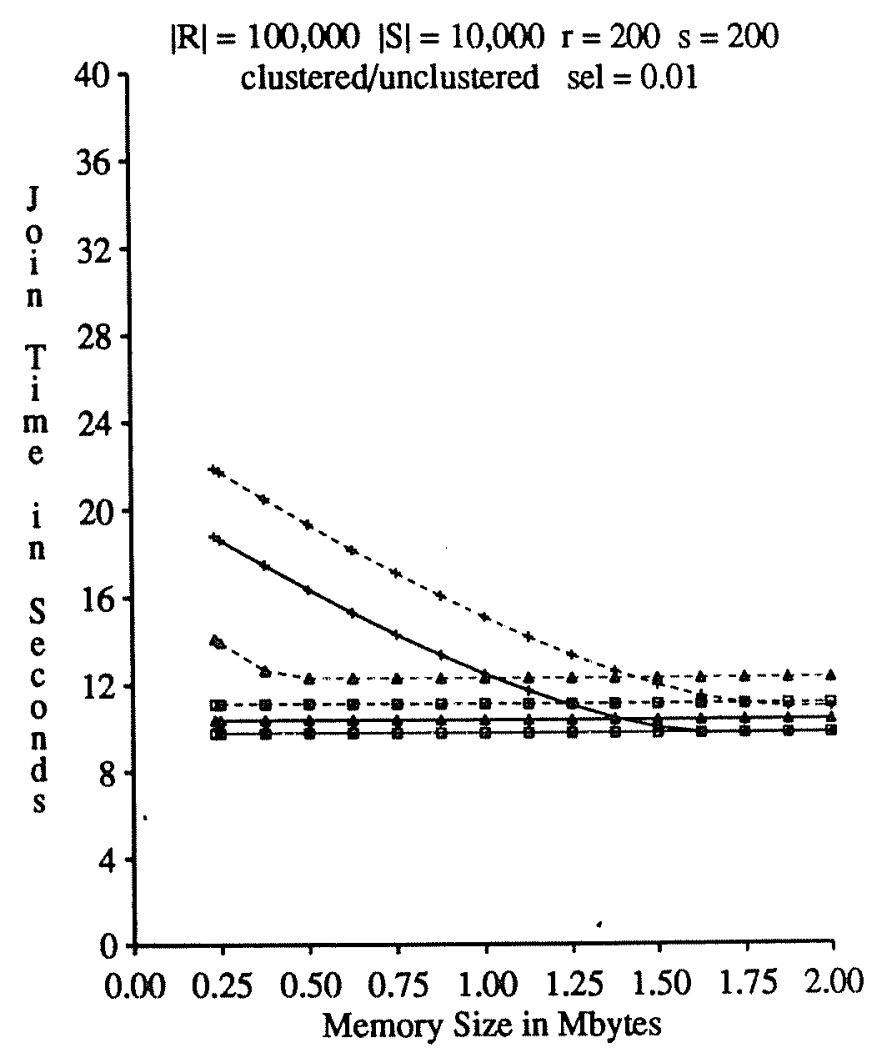

(F)

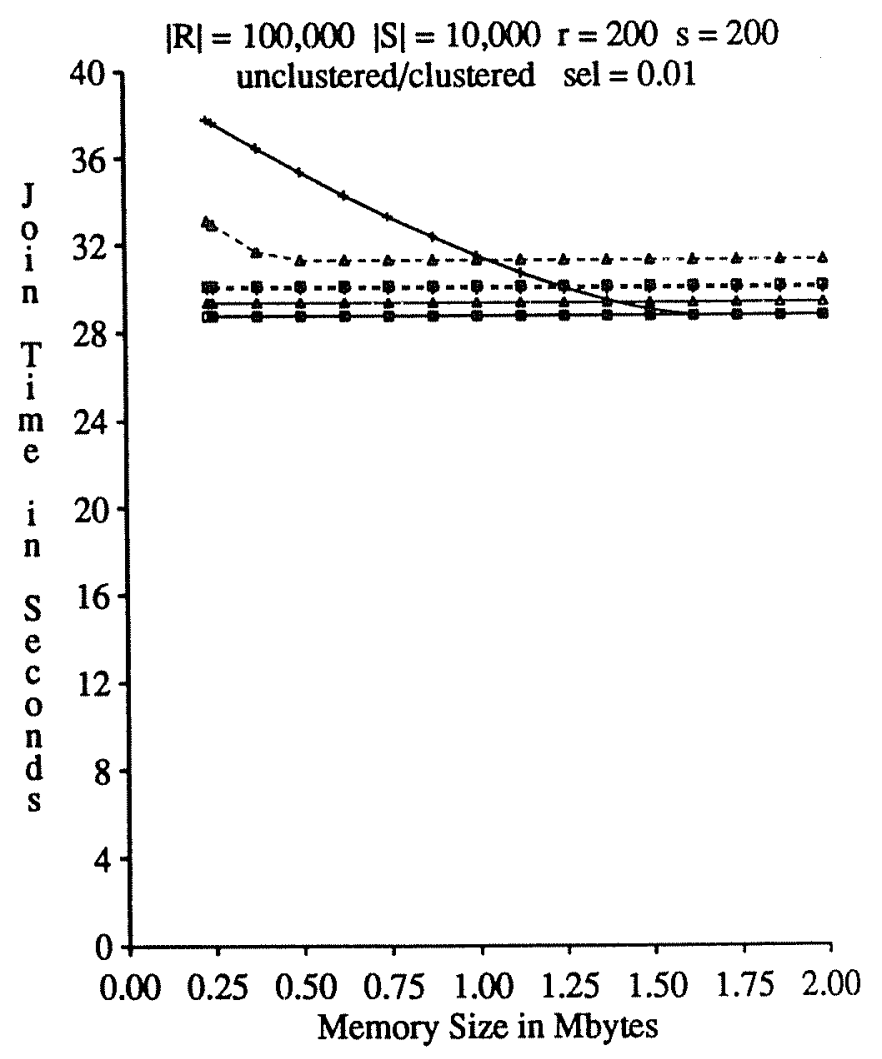

(H)

Figure 4: Results for Medium-Sized Joins 


\subsection{Performance Results for Small Joins}

The results for small joins are presented in Figure 5. Only the index-nested-loops algorithm and the pointerbased nested-loops algorithm are compared here because they perform as well as or better than the other algorithms in their respective classes on small joins. In all of the graphs, the size of memory was kept fixed at $1 / 4 \mathrm{Mbytes}$.

As indicated, the results in Figure 5 are shown in percentage terms. The total time to execute the join using the index-nested-loops algorithm was computed and the percentage difference between that time and the time to execute the pointer-based nested-loops algorithm was then plotted. The reason for displaying the results in this manner is to make the performance differences between the two algorithms clearer. This was not done for the previous results because they were not uniform enough to make this a viable approach.

The graphs in the top half of Figure 5 are for the clustered/unclustered index combination. Graph I shows the performance of the join algorithms when $\mathrm{R}$ is the same size as $\mathrm{S}$. As shown, the pointer-based nested-loops algorithm reduces the join time by almost $50 \%$ when more than 10 objects in $\mathrm{R}$ are joined with $\mathrm{S}$. In this case, the cost to execute the index-nested-loops algorithm is dominated by the $\mathrm{V} / \mathrm{O}$ cost to read the index on $\mathrm{S}$ and $\mathrm{S}$ itself, with roughly the same number of pages being read in each instance. Therefore, the cost to read the index on $S$ accounts for about one half of the cost to execute the index-nested-loops algorithm. Since the pointer-based nested-loops algorithm eliminates the cost to read the index on $\mathrm{S}$, it effectively reduces the cost of the join by $50 \%$.

Graph J shows the performance of the join algorithms when $\mathrm{R}$ is ten times the size of $\mathrm{S}$. As shown, the performance benefit of the pointer-based nested-loops algorithm decreases as the size of the join increases. In this case, the index on $S$ is small enough so that some of its leaf pages end up being referenced more than once by the index probes of the index-nested-loops algorithm. (Recall that in the index-nested-loops algorithm, a given leaf page $L_{i}$ may be referenced several times, but because the result of the selection on $\mathrm{R}$ is sorted by the join attribute, $L_{i}$ is guaranteed to be read from disk only once even if it is referenced several times.) In contrast to its index, however, $\mathrm{S}$ is still large enough in this case so that each probe of $S$ causes a different page in $S$ to be accessed. The net effect is that as the size of the join increases, the proportional cost of the index probes in the index-nested-loops algorithm decreases; this in turn causes the performance benefit of the pointer-based nested-loops algorithm to decrease somewhat.

Finally, the graphs for the unclustered/clustered index combination are presented in the bottom of Figure 5 . The differences between these graphs and the graphs in the upper half of Figure 5 are due to the fact that an unclustered index is used to read $R$. In this case, the cost to execute the index-nested-loops algorithm is dominated by the I/O cost to read $R$, the index on $S$, and $S$ itself, with roughly the same number of pages being read in each instance. Therefore, the cost to read the index on $S$ accounts for about one third of the cost to execute the index- 


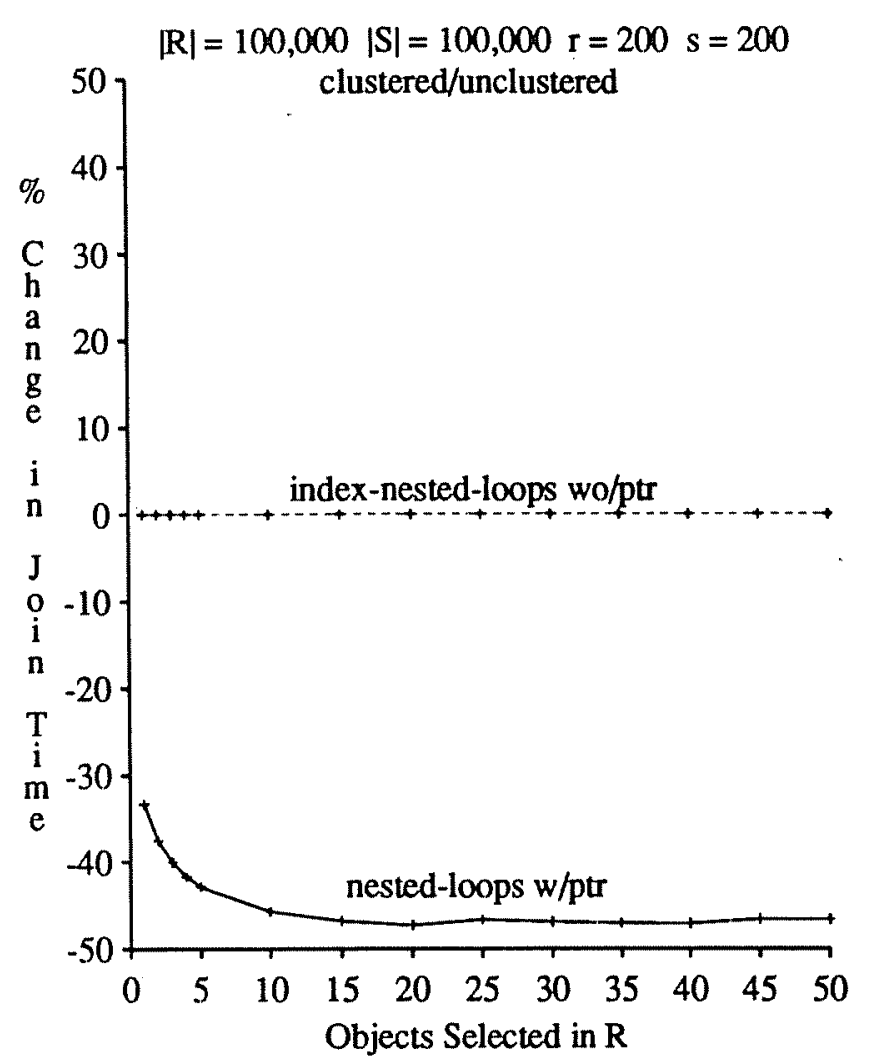

(I)

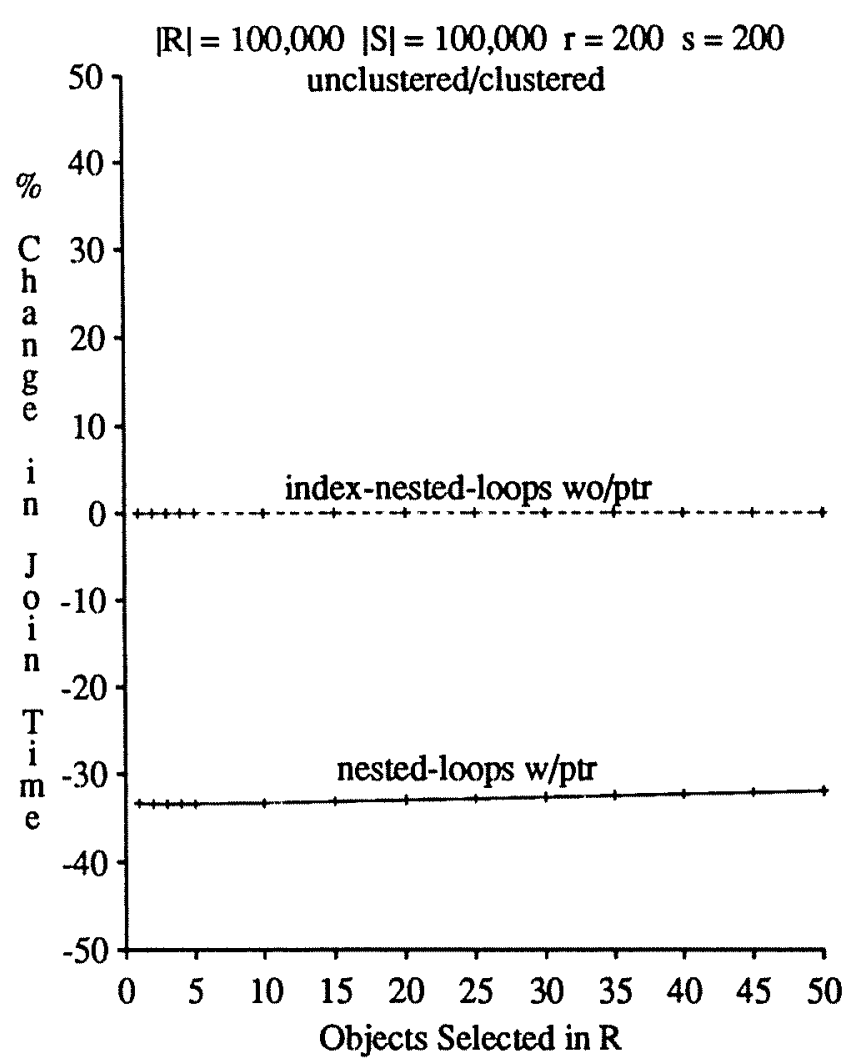

(K)

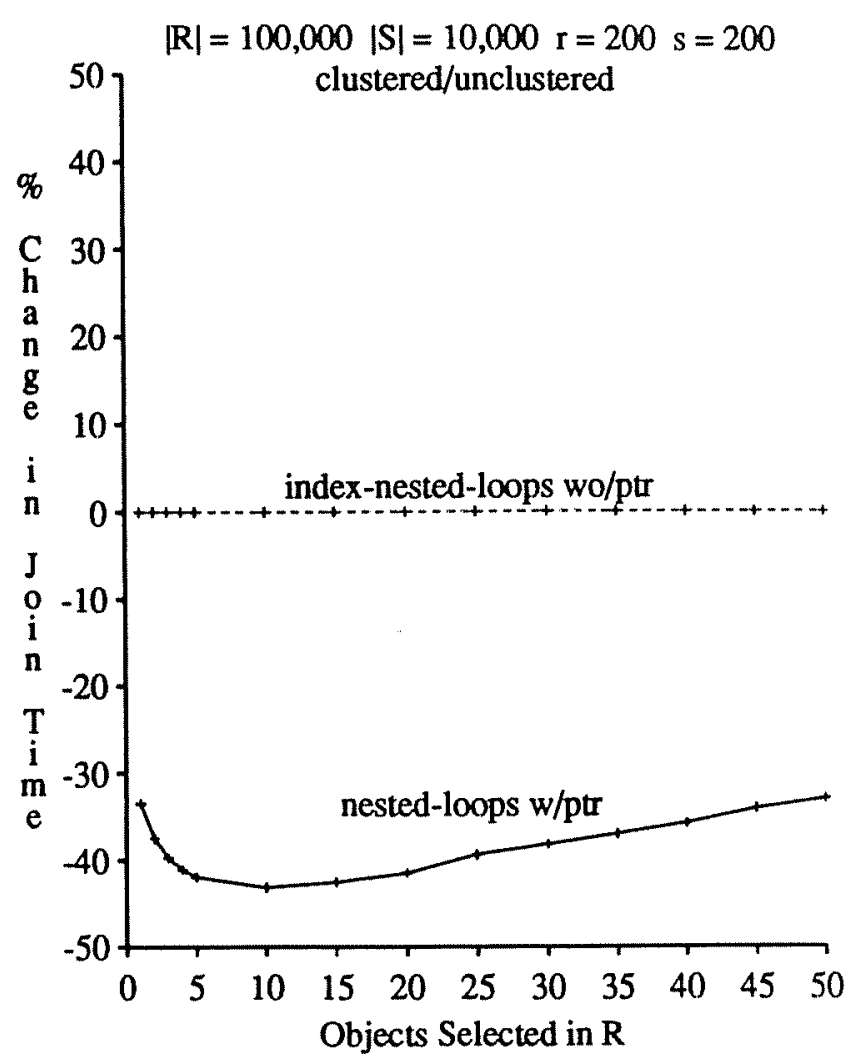

(J)

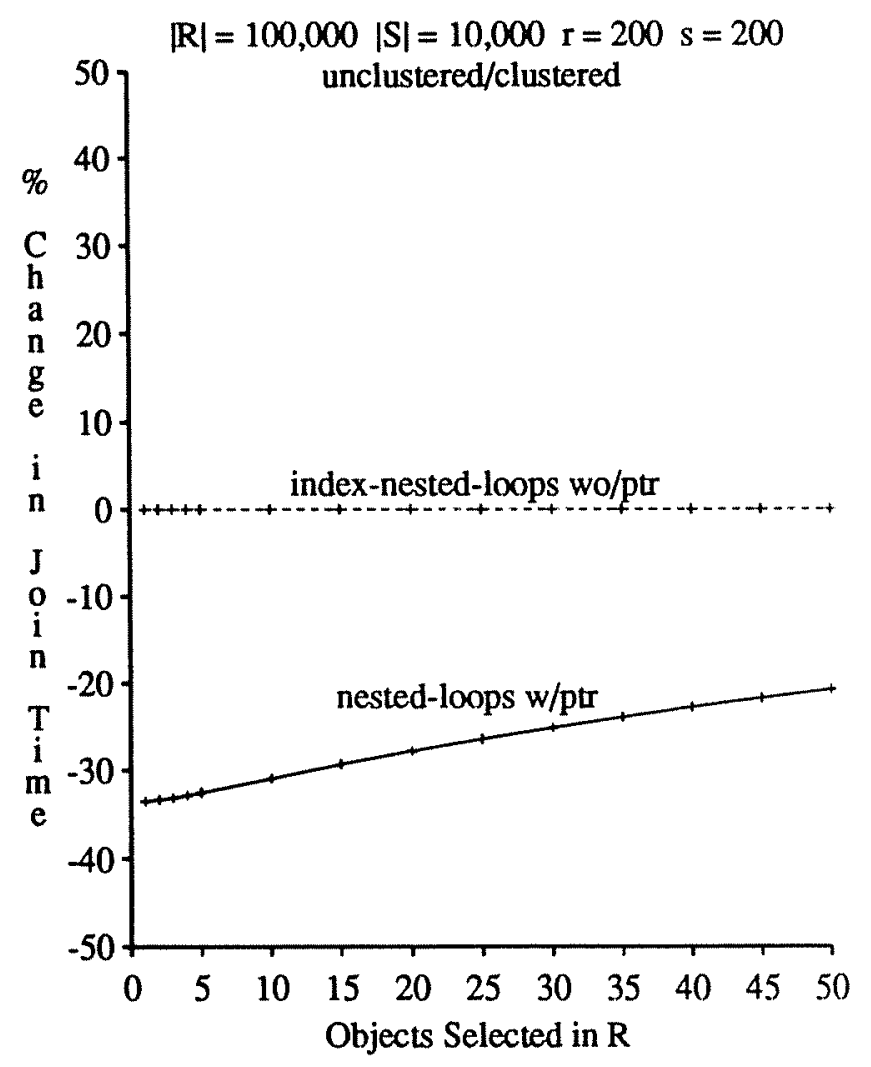

(I)

Figure 5: Results for Small Joins 
nested-loops algorithm. Since the pointer-based nested-loops algorithm eliminates the cost to read the index on $S$, it effectively reduces the cost of the join by $33 \%$. The line for the pointer-based nested-loops algorithm has an upward slope in Graph L for the same reason it has an upward slope in Graph J.

Once again, the graphs for the clustered/clustered and unclustered/unclustered index combinations have not been presented here. Although the graphs for those index combinations do not follow quite the same patterns as those in Figure 5, similar benefits and tradeoffs were observed; the pointer-based algorithms always outperformed their standard counterparts by $30 \%$ or more.

\section{TWO OTHER POINTER-BASED JOIN ALGORITHMS}

As mentioned earlier, a many-to-one pointer structure cannot be used effectively on select-project-join queries where $\mathrm{R}$ is joined to a selection on $\mathrm{S}$. To process such joins with pointers, a different kind of pointer structure is needed, where each object in $S$ is linked to the objects in $R$ that are related to it. One possibility is a one-to-many pointer structure, where each object in $S$ contains a set of embedded pointers linking that object to its related objects in R. Figure 6 illustrates this type of pointer structure. In this section, we outline two pointer-based join algorithms that use such a pointer structure.

Let $S^{\prime}$ be the result of the selection predicate on $S$. For the moment, assume that $S^{\prime}$ fits in memory. The first algorithm executes as follows:

(1) $S^{\prime}$ is read into memory page by page. After a page in $S^{\prime}$ has been read into memory, each object on that page is processed. When an object $s_{i}$ of $S^{\prime}$ is processed, the pointers stored in $s_{i}$ are hashed on their PID values, and the resulting hash values are used to populate a memory resident hash table. The hash table is structured in such a way that all the pointers which reference the same page in $R$ are grouped together in the same hash entry (see

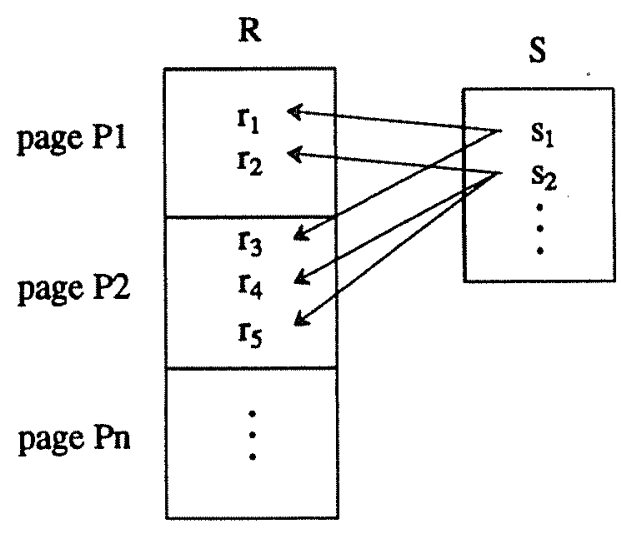

Figure 6: Example of On-To-Many Pointer Structure 
Figure 7).

(2) Let $r_{i}$.ptr be one of the pointers stored in $s_{i}$. When $r_{i}$.ptr is hashed on its PID value, a paired entry of the form $s_{i} . p t r, r_{i} . p t r$ is put in the hash table, where $s_{i} . p t r$ is a pointer to $s_{i}$ in memory and $r_{i} . p t r$ is a pointer to $r_{i}$ on disk. The version of $s_{i}$ that is stored in memory is optionally stripped of its pointers to $\mathrm{R}$ in order to conserve space.

(3) Once the hash table for $\mathrm{S}^{\prime}$ has been built, each of its hash chains is scanned. Each time a new hash entry $H$ is encountered on a chain, the page $P_{i}$ in R associated with $H$ is read, and the paired entries in $H$ are used to join the objects in $P_{i}$ with $\mathrm{S}^{\prime}$.

The above algorithm is analogous to the pointer-based hybrid-hash algorithm that was discussed earlier. It should be clear that algorithms which are analogous to the pointer-based nested-loops and sort-merge algorithm are also possible.

Note that the above algorithm is easily extended for the case when $S^{\prime}$ is larger than memory. This is accomplished by repeatedly executing steps (1)-(3) on memory-sized chunks of $S^{\prime}$ until all of $S^{\prime}$ has been joined with $R$. The resulting algorithm could be used to process full joins of $R$ and $S$, as well as joins with a selection predicate on S. Such an algorithm would be similar in nature to the one described in [Vald87], which uses a join index. The main difference here is that a runtime hash table is used to group pointer pairs rather than a pre-computed join index.

Unfortunately, the preceding algorithm in its extended form can cause each page of $R$ to be read more than once if $\mathrm{S}^{\prime}$ is bigger than memory. This is because there is no guarantee that all the pointers to a particular page of $\mathrm{R}$ will be contained within one memory-sized chunk of $S^{\prime}$. Consequently, for large joins, it may be more efficient to group objects of $S^{\prime}$ into partitions, much like in the pointer-based hybrid-hash algorithm that was discussed earlier. Doing so would ensure that each page of $R$ is read just once. An algorithm based on partitioning $S^{\prime}$ in such a

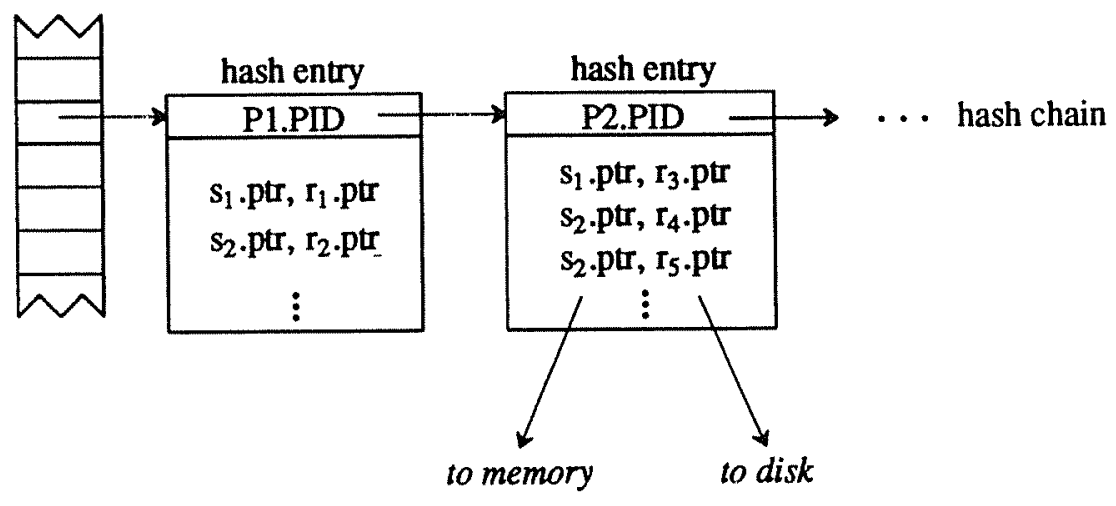

Figure 7: The Structure of the Hash Table 
manner is as follows:

(1) $S^{\prime}$ is read into memory and divided into $B+1$ partitions $S_{0}^{\prime}, S_{1}^{\prime}, \ldots S_{B}^{\prime}$, much like $\mathrm{R}$ was partitioned in the pointer-based hybrid-hash algorithm. The difference here is that every object $s$ in $S^{\prime}$ is hashed on each of the pointers that are stored in it. This in turn causes a copy of $s$ to be stored in each of the partitions to which one or more of its pointers hashes.

(2) Each partition of $S_{i}^{\prime}$ of $S^{\prime}$ is joined with $\mathrm{R}$ by taking $S_{i}^{\prime}$ and building a hash table for it in memory. The hash table for each $S_{i}^{\prime}$ has the same structure as the one depicted in Figure 7. The value of $B$ is chosen so that the hash table for each $S_{i}^{\prime}$ fits in memory and such that the hash table for $S_{0}^{\prime}$ can be built in memory as $S^{\prime}$ is partitioned.

(3) Once the hash table for $S_{i}{ }_{i}$ has been built, each of its hash chains are scanned as described in step (3) of the preceding algorithm to join $S_{i}^{\prime}$ with $\mathrm{R}$.

It remains to be determined under what conditions the above algorithm performs better than the one preceding it. For small joins, where $S^{\prime}$ fits in memory, the second algorithm degenerates into the first one. But when $S^{\prime}$ is much larger than memory, the second algorithm is likely to outperform the first one because of the way it optimizes its reads of $\mathrm{R}$. The analysis of each of the above algorithms and a comparison of their performance to standard join algorithms (with and without a join index) is left for future study.

\section{RELATED WORK}

Clearly, our work is related to the previous studies that have looked at the performance of different join algorithms, particularly [Blas77] and [Shap86]. In [Blas77], the nested-loops and sort-merge algorithms were described and analyzed, while in [Shap86], the hybrid-hash algorithm was analyzed and shown to be generally superior to the sort-merge as well as the Grace and simple-hash join algorithms. Both of these papers were concerned with joins in a purely relational context, however, and consequently neither considered pointer-based joins.

Perhaps the work that is most closely related to ours is that of [Vald87]. In that paper, auxiliary data structures called join indicies were described as a way to speedup join processing. If, for example, the join of relations $\mathrm{R}$ and $S$ was frequently needed, then a join index for $R$ and $S$ would be maintained. The join index would consist of pairs of pointers, matching each record in $\mathrm{R}$ with the record(s) it joins with in $\mathrm{S}$, and it would effectively implement the pre-computed join of $R$ and $S$.

In [Vald87], joins using a join index were shown to be superior to hybrid-hash joins in many situations. For the many-to-one types of joins we have analyzed here, however, it should be clear that our pointer-based algorithms will always outperform a join index. This is because the embedded pointers we have assumed effectively imple- 
ment a join index without the overhead of accessing (and maintaining) an auxiliary data structure.

\section{CONCLUSION}

This paper described three pointer-based join algorithms that are simple variants of the standard nested-loops, sort-merge, and hybrid-hash join algorithms used in relational database systems. We showed that, given the appropriate pointer structures, some common types of joins in relational and object-oriented database systems can be executed using our pointer-based algorithms.

For much of the paper, an analysis was carried out to compare the pointer-based algorithms to their standard, non-pointer-based counterparts. In addition to providing a basis for comparison, the cost equations that were derived in the analysis can also be used in query optimization and physical database design. In the analysis, the join of two sets $\mathrm{R}$ and $\mathrm{S}$ in a many-to-one relationship was studied. A simple pointer structure was assumed, where each object in R contained a pointer to its related object in S. Two types of joins were analyzed: full joins of R and S, and small to medium-sized joins with a selection predicate on $\mathbf{R}$.

For full joins, the results of the analysis showed that the pointer-based sort-merge and hybrid-hash join algorithms can provide savings of $30 \%$ or more when the size of $\mathrm{R}$ (in pages) is roughly the same size as $\mathrm{S}$ or smaller. These results are due to the fact that $\mathrm{S}$ does not need to be sorted or partitioned in the pointer-based algorithms. The results also showed, however, that the pointer-based algorithms do not perform as well as the standard hybrid-hash algorithm when the size of $\mathrm{R}$ is significantly larger than the size of $\mathrm{S}$. Moreover, the pointer-based nested-loops join algorithm was shown to perform very poorly in almost all cases. These negative results are important because they show that it is unwise for object-oriented database systems to support only pointer-based join algorithms. They also show that to make effective use of pointers, something more intelligent than a nested-loops approach (i.e., naive pointer traversal) is needed for high performance on large joins.

For medium-sized joins, where $1 \%$ of the objects in $\mathrm{R}$ were joined with $\mathrm{S}$, the results showed that the pointer-based sort-merge and hybrid-hash algorithms always outperformed their standard counterparts, providing gains of up to $30 \%$ in many cases. The pointer-based nested-loops algorithm was again shown to perform poorly, although not in all cases. Finally, for small joins, where $0.01 \%$ of the objects in R were joined with $\mathrm{S}$, all of the pointer-based join algorithms outperformed their standard counterparts by $30 \%$ or more. Both of these results are largely due to the fact that index lookups on $S$ are eliminated in the pointer-based algorithms. The conclusion to be drawn here is that, for small to medium-sized joins, which are probably a very common type of join, pointer-based join algorithms can provide significant performance gains.

In addition to the pointer-based joins that were analyzed, we also described two other pointer-based join algorithms that make use of a one-to-many pointer structure; that is, a pointer structure in which each object in $S$ 
contains a set of embedded pointers linking that object to its related objects in $\mathrm{R}$. The performance analysis of those algorithms, which are both based on hashing, will be addressed in future work.

As far as implementation results go, we are currently in the process of obtaining experimental results for some of the join algorithms described here using the incremental join facility of Starburst [Care90, Haas90]. That facility maintains the types of pointer structures that our algorithms require and was in fact largely designed and implemented to study the potential benefits of pointer-based joins. The experiments being conducted, which are described in [Care90], compare a variety of pointer-based join algorithms to their standard counterparts under three different clustering strategies. 


\section{APPENDIX:}

\section{Analysis of Small To Medium-Sized Joins with the Unclustered/Clustered Index Combination}

In this appendix, small to medium-sized joins with the unclustered/clustered index combination are analyzed. Much of the analysis follows directly from that presented in Section 4.4, and the notation used here is the same as the notation that was used earlier.

\section{Standard Index-Nested-Loops}

As one would expect, the analysis for the index-nested-loops algorithm with the unclustered/clustered index combination is similar to the analysis that was presented earlier for that algorithm in Section 4.4.1. Here, the analysis proceeds by noting that the cost to read the index on $R$, as opposed to $R$ itself, is the same as in the earlier analysis. This follows because the cost to read the index alone is the same whether it is a clustered or unclustered index. Furthermore, the cost to probe the index on $S$ is also the same as before due to the fact that $R$ and $S$ are always assumed to be relatively unclustered. As a result of these observations, the only real differences from the earlier analysis are the cost to read $R^{\prime}$ (the result of the selection on $R$ ) and the cost to access $S$.

A precise estimate of the cost to read $R^{\prime}$ can be derived by once again making use of the Yao function $Y($ ). However, because the index on $R$ is an unclustered index, and because the number of objects in $R^{\prime}$ is small relative to $\mathrm{R}$ in the examples that we analyze, we can safely assume that each object in $\mathrm{R}^{\prime}$ resides on a different page in $\mathrm{R}$. As a result, the cost to read $\mathrm{R}^{\prime}$ is approximately $\left|\mathrm{R}^{\prime}\right| \cdot I O$.

To determine the cost to access $S$, recall that the index-nested-loops algorithm sorts the objects in $R^{\prime}$ by their join attribute before the index on $S$ is probed. Because the index on $S$ is a clustered index, this ensures that each page in $S$ will be accessed only once. Therefore, the cost to access $S$ can be calculated by determining how many pages in S participate in the join, which can be determined using the same analysis that was used in Section 4.4.5. Applying that analysis again, the cost to access $S$ is:

$$
P_{s} \cdot Y\left(|\mathrm{R}|, k \cdot O_{s,}\left|\mathrm{R}^{\prime}\right|\right) \cdot I O
$$

Based on the above analysis, and based on the analysis for the index-nested-loops algorithm with the clustered/unclustered index combination, the cost of the index-nested-loops algorithm with the unclustered/clustered index combination is: 
$2 \cdot I O+2 \cdot \log _{2} b \cdot c o m p a r e$

$+\left\lceil\left|R^{\prime}\right| / b-1\right\rceil \cdot I O$

$+\left|\mathbf{R}^{\prime}\right| \cdot I O$

$+\left|R^{\prime}\right| \cdot$ move $_{r}$

$+\left|R^{\prime}\right| \cdot \log _{2}\left|R^{\prime}\right| \cdot\left(\right.$ compare + swap $\left._{r}\right)$

$+10$

$+2 \cdot\left|\mathrm{R}^{\prime}\right| \cdot \log _{2} b \cdot$ compare

$+\lceil|S| / b\rceil \cdot Y\left(|\mathrm{R}|, b \cdot k,\left|\mathrm{R}^{\prime}\right|\right) \cdot I O$

$+P_{s} \cdot Y\left(|\mathrm{R}|, k \cdot O_{s},\left|\mathrm{R}^{\prime}\right|\right) \cdot I O$ descend the index on $\mathbf{R}$

scan across the leaves of the index on $R$ read $R^{\prime}$

extract $R^{\prime}$ from $R$ in memory before sorting cost to sort $R^{\prime}$

read the root page of the index on $S$

probe the index on $S$ for each object in $R^{\prime}$

access the leaf pages of the index on $S$

access $\mathrm{S}$

\section{The Remaining Algorithms}

The analysis for the remaining algorithms is straightforward. For each algorithm, it should be clear that the cost to read the index on $R$ and the cost to read $R^{\prime}$ is the same as in the preceding analysis. Furthermore, the cost to read $S$ in each algorithm is the same as in Section 4.4 because $R$ and $S$ are always assumed to be relatively unclustered. Finally, none of the remaining algorithms use the index on $S$, so that cost does not need to be considered. Based on these observations, the cost of each of the remaining algorithms with the clustered/unclustered index combination can be obtained by taking the analysis for each algorithm as presented in Section 4.4 and changing the cost to read $\mathrm{R}^{\prime}$ to be $\left|\mathrm{R}^{\prime}\right| \cdot \mid O$. 


\section{REFERENCES}

[Blas77] M. Blasgen and K. Eswaran, "Storage and Access in Relational Databases," IBM Syst. Journal, 16(4), 1977.

[Care88] M. Carey et al., "A Data Model and Query Language for EXODUS," Proc. of the 1988 ACM-SIGMOD Conf., Chicago, IL, 1988.

[Care89] M. Carey et al, "The EXODUS Extensible DBMS Project: An Overview," in Readings in ObjectOriented Databases, S. Zdonik and D. Maier, eds., Morgan-Kaufman Publ. Co., 1989.

[Care90] M. Carey et al, "An Incremental Join Attachment for Starburst," submitted for publication.

[Chan82] A. Chan, "Storage and Access Structures to Support a Semantic Data Model," Proc. of the 1982 VLDB Conf., Mexico City, Mexico, 1982.

[Chou85] H-T. Chou and D. Dewitt, "An Evaluation of Buffer Management Strategies for Relational Database Systems," Proc. of the 1985 VLDB Conf., Stockholm, Sweden, Aug. 1985.

[Date86] C. Date, "An Introduction to Database Systems," Ch. 3., pg. 56, Addison-Wesley, Reading Mass. 1986.

[Dewi85] D. DeWitt and R. Gerber, "Multiprocessor Hash-Based Join Algorithms," Proc. of the 1985 VLDB Conf., Stockholm, Sweden, Aug. 1985.

[Deaux90] O. Deux et al, "The Story of O2," IEEE Trans. on Knowledge and Data Eng., March 1990.

[Haas90] L. Haas et al, "Starburst Mid-Flight: As the Dust Clears," IEEE Trans. on Knowledge and Data Engineering, March 1990.

[Horn87] M. Hornick and S. Zdonik, "A Shared, Segmented Memory System for an Object-Oriented Database," ACM Trans. on Office Info. Sys. 5(1), Jan. 1987.

[Kim89] W. Kim, "A Model of Queries for Object-Oriented Databases," Proc. of the 1989 VLDB Conf., Amsterdam, The Netherlands, Aug. 1989.

[Knut73] D. Knuth, The Art of Computer Programming: Sorting and Searching, Vol. 3, Addison-Wesley, Reading, Mass., pg. 254, 1973.

[Mack86] L. Mackert and G. Lohman, "R* Optimizer Validation and Performance Evaluation for Local Queries," Proc. of the ACM-SIGMOD Conf., Washington, D.C, 1986.

[Mack89] L. Mackert and G. Lohman, "Index Scans Using a Finite LRU Buffer: A Validated I/O Model," $A C M$ Trans. on Database Systems 14(3), Sept. 1989.

[Seve76] D. Severance and G. Lohman, "Differential Files: Their Application to the Maintenance of Large Databases," ACM Trans. on Database Systems 1(3), Sept. 1976.

[Shap86] L. Shapiro, "Join Processing in Database Systems with Large Main Memories," ACM Trans. on Database Systems 11(3), Sept. 1986.

[Shek88] E. Shekita et al., An Introduction to the EXODUS Storage Manager, user documentation from the EXODUS Extensible DBMS Project, Univ. of Wisconsin, Madison, May 1988.

[Ship81] D. Shipman, "The Functional Data Model and the Data Language DAPLEX," ACM Trans. on Database Sys. 6(1), Sept. 1987.

[Vald87] P. Valduriez, "Join Indices," ACM Trans. on Database Systems 12(2), June 1987.

[Vele89] F Velez et al, "The O2 Object Manager: An Overview," Proc. 1989 VLDB Conf., Amsterdam, The Netherlands, Aug. 1989.

[Yao77] S. Yao, "Approximating Block Accesses in Database Organizations," Comm. of the ACM 20(4), April 1977.

[Zani83] C. Zaniolo, "The Database Language GEM," Proc. of the ACM-SIGMOD Conf., San Jose, CA, 1983. 
